\title{
SGAS 143845.1+145407: A BIG, COOL STARBURST AT REDSHIFT 0.816*
}

\author{
Michael D. Gladders ${ }^{1,2}$, Jane R. Rigby ${ }^{3}$, Keren Sharon ${ }^{2}$, Eva Wuyts ${ }^{1,2}$, Louis E. Abramson ${ }^{1,2}$, HÅ Kon Dahle ${ }^{4}$, \\ S. E. Persson ${ }^{5}$, Andrew J. Monson ${ }^{5}$, Daniel D. Kelson ${ }^{5}$, Dominic J. Benford ${ }^{3}$, David Murphy ${ }^{5}$, Matthew B. Bayliss ${ }^{6,7}$, \\ Keely D. Finkelstein ${ }^{8}$, Benjamin P. Koester ${ }^{9}$, Alissa Bans ${ }^{1}$, ERic J. BaXter ${ }^{1}$, And Jennifer E. Helsby ${ }^{1,2}$ \\ ${ }^{1}$ Department of Astronomy \& Astrophysics, The University of Chicago, 5640 South Ellis Avenue, \\ Chicago, IL 60637, USA; gladders@oddjob.uchicago.edu \\ ${ }^{2}$ Kavli Institute for Cosmological Physics at the University of Chicago, Chicago, IL, USA \\ ${ }^{3}$ Observational Cosmology Lab, NASA Goddard Space Flight Center, Greenbelt, MD 20771, USA \\ ${ }^{4}$ Institute of Theoretical Astrophysics, University of Oslo, P.O. Box 1029, Blindern, NO-0315 Oslo, Norway \\ ${ }^{5}$ Carnegie Observatories, 813 Santa Barbara Street, Pasadena, CA 91101, USA \\ ${ }^{6}$ Harvard-Smithsonian Center for Astrophysics, 60 Garden Street, Cambridge, MA 02138, USA \\ ${ }^{7}$ Department of Physics, Harvard University, 17 Oxford Street, Cambridge, MA 02138, USA \\ ${ }^{8}$ Department of Astronomy, University of Texas at Austin, 2515 Speedway Stop C1400, Austin, TX 78712, USA \\ ${ }^{9}$ Physics Department, University of Michigan, Ann Arbor, MI 48109, USA \\ Received 2012 May 10; accepted 2012 December 23; published 2013 February 5
}

\begin{abstract}
We present the discovery and detailed multi-wavelength study of a strongly lensed luminous infrared galaxy at $z=$ 0.816. Unlike most known lensed galaxies discovered at optical or near-infrared wavelengths, this lensed source is red, $\left(r-K_{S}\right)_{A B}=3.9$, which the data presented here demonstrate is due to ongoing dusty star formation. The overall lensing magnification (a factor of 17) facilitates observations from the blue optical through to $500 \mu \mathrm{m}$, fully capturing both the stellar photospheric emission and the re-processed thermal dust emission. We also present optical and near-IR spectroscopy. These extensive data show that this lensed galaxy is in many ways typical of IR-detected sources at $z \sim 1$, with both a total luminosity and size in accordance with other (albeit much less detailed) measurements for samples of galaxies observed in deep fields with the Spitzer telescope. Its far-infrared spectral energy distribution is well fit by local templates that are an order of magnitude less luminous than the lensed galaxy; local templates of comparable luminosity are too hot to fit. Its size ( $D \sim 7 \mathrm{kpc})$ is much larger than local luminous infrared galaxies, but in line with sizes observed for such galaxies at $z \sim 1$. The star formation appears uniform across this spatial scale. In this source, the luminosity of which is typical of sources that dominate the cosmic infrared background, we find that star formation is spatially extended and well organized, quite unlike the compact merger-driven starbursts that are typical for sources of this luminosity at $z \sim 0$.
\end{abstract}

Key words: galaxies: evolution - galaxies: high-redshift - gravitational lensing: strong

Online-only material: color figures

\section{INTRODUCTION}

Strongly gravitationally lensed galaxies offer a unique view of the distant universe by providing both a surfeit of photons and access to physical scales not resolvable when studying galaxies that have not been lensed. Furthermore, lensed galaxies are likely to probe a more typical portion of the luminosity function (e.g., Bayliss et al. 2010) than strictly magnitude-limited blank field studies. The typical bright lensed source, from samples selected at optical wavelengths, is a vigorously star-forming galaxy at $z \sim 2$ (Bayliss et al. 2011; Bayliss 2012) with blue colors and modest reddening (Wuyts et al. 2012). The brightest of these lensed sources, which are typically magnified by factors of 20 or more (e.g., Williams \& Lewis 1996; Allam et al. 2007; Sharon et al. 2012), are of particular interest in part because they enable studies in wavelength regimes where current facilities lack the sensitivity to reach high-redshift galaxies (see the discussion in Siana et al. 2009). The brightest lensed sources reported to date from discoveries at optical wavelengths are fairly blue, from the prototype MS1512-cB58 (Yee et al. 1996)

\footnotetext{
* This paper includes data gathered with the $6.5 \mathrm{~m}$ Magellan Telescopes located at Las Campanas Observatory, Chile. This paper is also based on observations made with the Nordic Optical Telescope, operated on the island of La Palma jointly by Denmark, Finland, Iceland, Norway, and Sweden, in the Spanish Observatorio del Roque de los Muchachos of the Instituto de Astrofísica de Canarias.
}

to more recent discoveries (e.g., Belokurov et al. 2007; Lin et al. 2009; Koester et al. 2010). The reddest of these is the Cosmic Eye at $z=3.074$ (Smail et al. 2007; Siana et al. 2009).

In the submillimeter, gravitational lensing in the weak limit has provided a sensitivity boost for submillimeter galaxy surveys (e.g., Smail et al. 1997; Egami et al. 2010), but these sources are typically magnified only by tens of percent, with no multiple imaging. As such, until recently, there were only a small number of robustly confirmed gravitationally lensed submillimeter galaxies with large magnifications (Borys et al. 2004; Kneib et al. 2004; Knudsen et al. 2009; Swinbank et al. 2010; Lestrada et al. 2011; Frayer et al. 2011; Combes et al. 2012; Fu et al. 2012). Expectations are that the new generation of millimeter and submillimeter telescopes should detect large numbers of lensed galaxies at these wavelengths (e.g., Herschel: Negrello et al. 2010; González-Nuevo et al. 2012; the South Pole Telescope: Vieira et al. 2010) and samples of lensed sources are now emerging from those efforts (e.g., Greve et al. 2012; Harris et al. 2012; Wardlow et al. 2012).

In this paper, we report the discovery of an unusually red and bright lensed galaxy, at a redshift of $z=0.816$. This galaxy is multiply imaged and magnified by a total factor of $16.8_{-6.2}^{+5.2}$ by a foreground galaxy cluster at $z=0.237$. We present photometric measurements of the source from the restframe ultraviolet to the far infrared, as well as both optical and near-infrared spectroscopy. From these data, we construct 


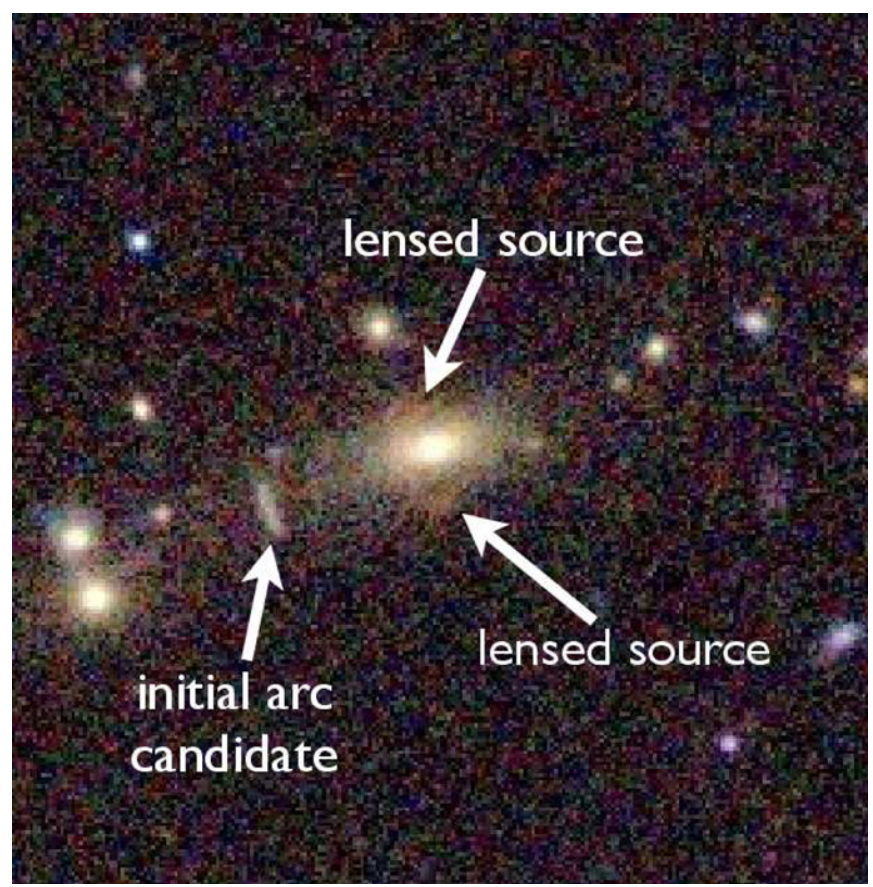

Figure 1. 90" $\times 90^{\prime \prime}$ image cutout from SDSS DR7 imaging data (blue: $g$ band, green: $r$ band, red: $i$ band $+z$ band). The faint red light around the BCG, which is barely visible all but to the right of the BCG, is the lensed source discussed in this paper. The arclet to the lower left is the candidate lensed source discussed in the Appendix.

(A color version of this figure is available in the online journal.)

a lens model, which we use to invert measurements to unlensed quantities in the source plane when required. These data show that the source is a luminous infrared galaxy (an LIRG) that is typical of star-forming galaxies at that epoch (Lagache et al. 2005; Le Floc'h et al. 2005; Caputi et al. 2007; Pascale et al. 2009). Section 2 describes how the lensed source was discovered as part of a larger effort to find and characterize lensed galaxies. Section 3 describes the follow-up observations, as well as the basic measurements of the source. Section 4 details the lens model and properties of the lensed source in the image plane; these measurements are discussed and contexualized in Section 5.

Throughout the paper, we adopt a flat cosmology with $\Omega_{M}=0.3$ and $H_{0}=70 \mathrm{~km} \mathrm{~s}^{-1} \mathrm{Mpc}^{-1}$. All magnitudes are reported on the $\mathrm{AB}$ system unless otherwise noted. All on-sky images show north as up and east to the right.

\section{DISCOVERY OF A RED HERRING AND A RED LENSED GALAXY}

The cluster SDSSJ $1438+1454$ was selected as a possible strong lensing system (M. D. Gladders et al., in preparation) from SDSS imaging. As described in the Appendix, this initial selection was likely due to the presence of a blue arclet feature 18 ".5 from the brightest cluster galaxy (BCG), with color, geometry with respect to the BCG, and a hint of curvature all suggestive of lensing, as shown in Figure 1. The Appendix also includes follow-up spectroscopy from the ARC $3.5 \mathrm{~m}$ that shows that this initial arc candidate is not lensed, but is rather a cluster galaxy at $z=0.231$.

However, another arc-like feature, this one faint and redder than the BCG, was just visible in the SDSS imaging. We had obtained warm Spitzer observations with IRAC at 3.6 and $4.5 \mu \mathrm{m}$ for a larger sample of $\sim 100$ lensing systems through

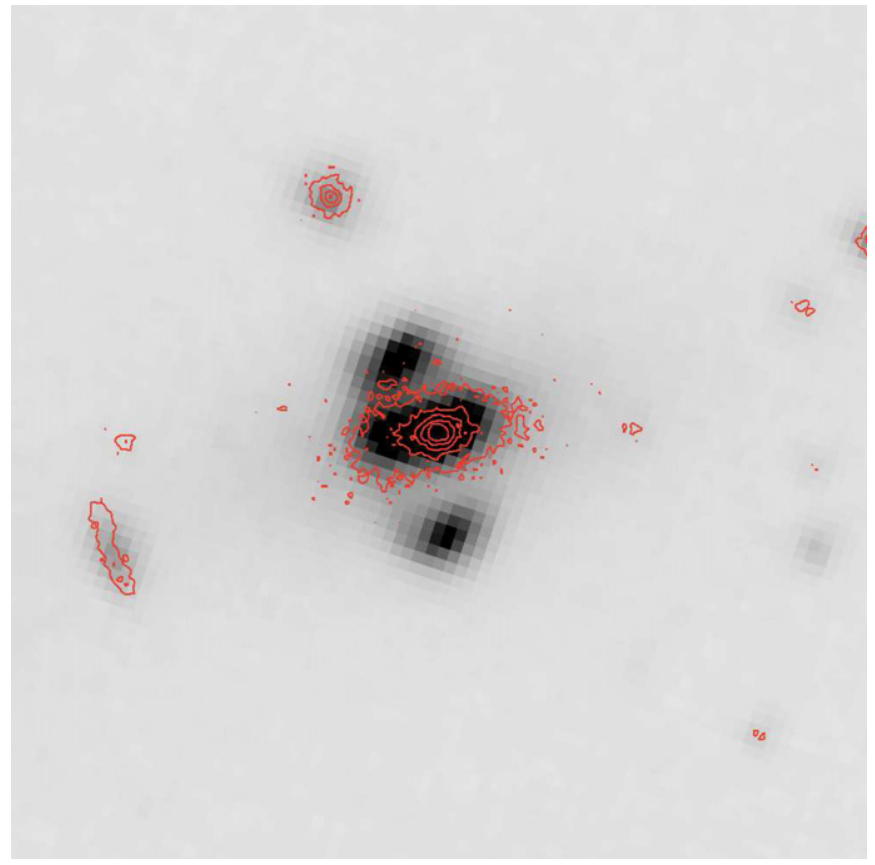

Figure 2. $45 \times 45^{\prime \prime}$ cutout from the Spitzer IRAC $3.6 \mu \mathrm{m}$ image (grayscale), with the NOT $g$-band image overplotted (red contours). Two extremely red sources above and below the BCG are immediately apparent; these are two images of SGAS 1438 .

(A color version of this figure is available in the online journal.)

Spitzer program 70154 (PI: M. D. Gladders). This Spitzer imaging (Figure 2 and Table 1) clearly showed that this source was infrared bright, very red in the $g-3.6 \mu \mathrm{m}$ and $g-4.5 \mu \mathrm{m}$ colors, and likely to be a multiply imaged lensed galaxy. This source is the subject of this paper. Following the naming conventions established in Koester et al. (2010), we refer to the lensing cluster as SDSSJ 1438+1454 and the lensed source as SGAS 143845.1+145407 (SGAS 1438 in short hereafter). The source name coordinates are derived from the position of the most isolated complete image, which is 5".6 almost due south of the BCG.

\section{FURTHER DATA AND MEASUREMENTS}

\subsection{Optical and Near-IR Imaging}

Motivated by the identification of SGAS $143845.1+145407$ as a possible lensed source from the SDSS and Spitzer imaging, we observed the field with other facilities. Near-IR imaging in the $Y J H$ and $K_{s}$ bands was acquired during commissioning of the FourStar camera at the Magellan Baade $6.5 \mathrm{~m}$ in early 2011. These near-IR images robustly confirmed that this was indeed a lensing geometry, as shown in Figure 3, and further demonstrated that the lensed source is extremely red. The FourStar $J H$ and $K_{s}$ images were flux-calibrated directly to the Two Micron All Sky Survey point-source catalog (Skrutskie et al. 2006), which is possible because the field of view of FourStar is large; calibration uncertainties are a few percent in these bands. The $Y$-band image was calibrated using a standard star observation ${ }^{10}$ acquired immediately prior to the science observation at a similar airmass. This calibration agrees with repeated $Y$-band calibrations of the instrument over months of

\footnotetext{
10 Calibrations of the $Y$-band and other medium-band filters available in FourStar have been undertaken in support of the Z-FOURGE project (Spitler et al. 2011) and will be published in detail elsewhere.
} 


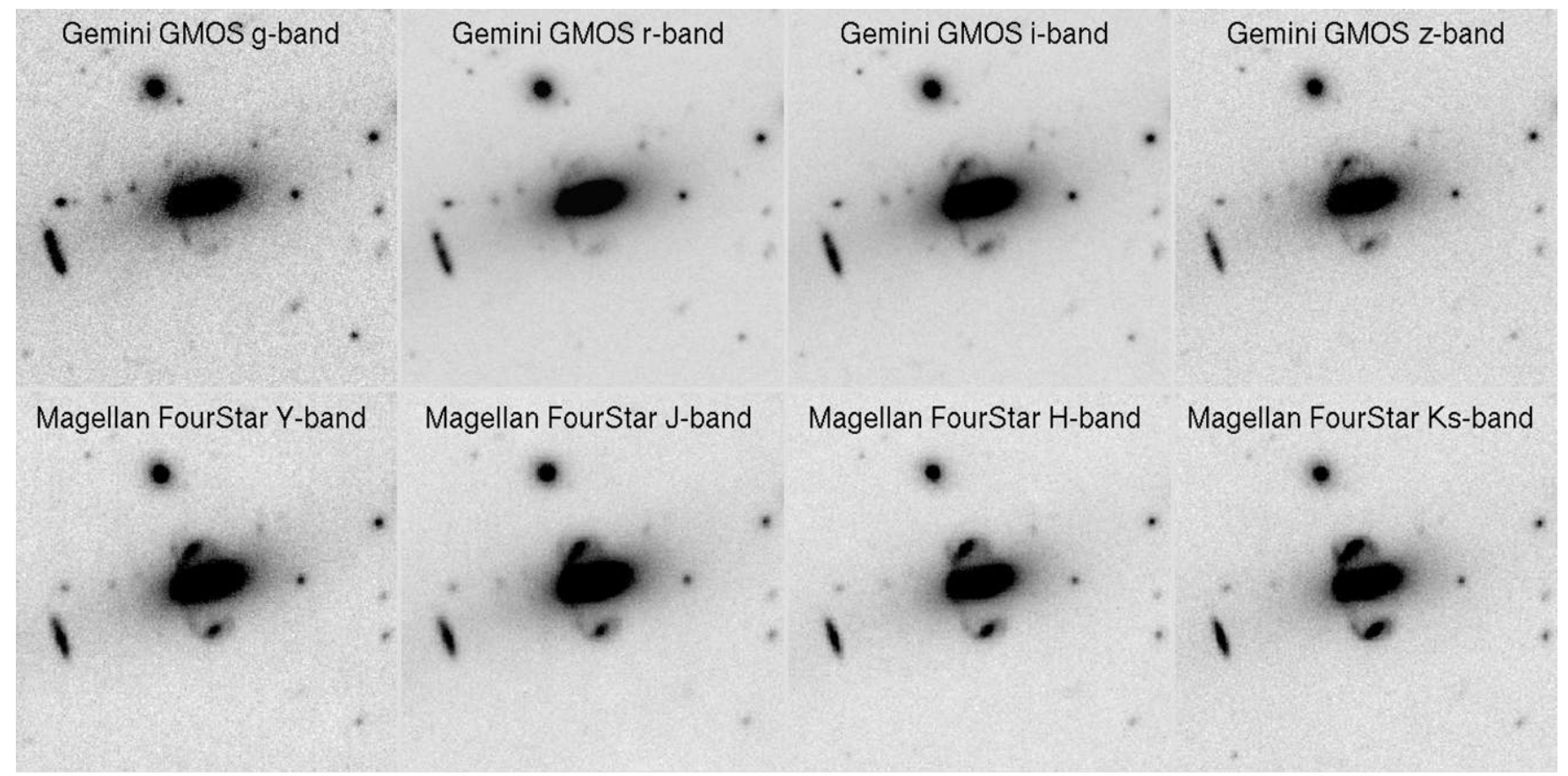

Figure 3. $45 \times 45^{\prime \prime}$ cutouts in the griz YJH and $K_{s}$ bands. All images have been scaled to approximately the same grayscale level for the BCG. Note the obvious multiplicity of images, particularly in the near-IR, which confirms the lensing interpretation.

Table 1

Imaging Observations and Photometry of SGAS 1438

\begin{tabular}{|c|c|c|c|c|c|}
\hline Telescope and Instrument & Filter & $\begin{array}{l}t_{\text {int }} \\
\text { (s) }\end{array}$ & $\begin{array}{c}\text { PSF } \\
\left({ }^{\prime \prime}\right)\end{array}$ & Date & $m_{\mathrm{AB}}$ \\
\hline Gemini-N GMOS & SDSS $g$ & 300 & 0.61 & 2011 Apr 29 & $21.45 \pm 0.16$ \\
\hline Gemini-N GMOS & SDSS $r$ & 300 & 0.56 & 2011 Apr 29 & $20.24 \pm 0.11$ \\
\hline Gemini-N GMOS & SDSS $i$ & 300 & 0.54 & 2011 Apr 29 & $18.85 \pm 0.10$ \\
\hline Gemini-N GMOS & $\operatorname{SDSS} z$ & 300 & 0.50 & 2011 Apr 29 & $18.10 \pm 0.09$ \\
\hline Magellan FourStar & $Y$ & 800 & 0.68 & 2011 Feb 19 & $18.03 \pm 0.12$ \\
\hline Magellan FourStar & $J$ & 800 & 0.66 & 2011 Feb 19 & $17.34 \pm 0.11$ \\
\hline Magellan FourStar & $H$ & 800 & 0.63 & 2011 Feb 19 & $16.84 \pm 0.11$ \\
\hline Magellan FourStar & $K_{s}$ & 1572 & 0.53 & 2011 Jan 23 & $16.31 \pm 0.11$ \\
\hline Spitzer IRAC ${ }^{\mathrm{a}}$ & $3.6 \mu \mathrm{m}$ & 600 & 1.7 & 2010 Sep 19 & $15.74 \pm 0.13$ \\
\hline Spitzer IRAC ${ }^{\mathrm{a}}$ & $4.5 \mu \mathrm{m}$ & 600 & 1.7 & 2010 Sep 19 & $16.14 \pm 0.13$ \\
\hline WISE & $12 \mu \mathrm{m}$ & 167 & 6.5 & $2010 \mathrm{Jan} / \mathrm{Jul}$ & $14.58 \pm 0.08^{\mathrm{c}}$ \\
\hline WISE & $22 \mu \mathrm{m}$ & 193 & 12.0 & $2010 \mathrm{Jan} / \mathrm{Jul}$ & $14.16 \pm 0.21^{\mathrm{c}}$ \\
\hline Herschel PACS ${ }^{b}$ & $70 \mu \mathrm{m}$ & 900 & 5.6 & $2011 \mathrm{Jul} 9$ & $12.06 \pm 0.15$ \\
\hline Herschel PACS ${ }^{b}$ & $100 \mu \mathrm{m}$ & 900 & 6.8 & $2011 \mathrm{Jul} 9$ & $11.11 \pm 0.15$ \\
\hline Herschel PACS ${ }^{b}$ & $160 \mu \mathrm{m}$ & 1800 & 11 & 2011 Jul 9 & $10.00 \pm 0.15$ \\
\hline Herschel SPIRE ${ }^{b}$ & $250 \mu \mathrm{m}$ & 185 & 18 & $2011 \mathrm{Jul} 12$ & $10.06 \pm 0.15$ \\
\hline Herschel SPIRE ${ }^{b}$ & $350 \mu \mathrm{m}$ & 185 & 25 & 2011 Jul 12 & $10.41 \pm 0.15$ \\
\hline Herschel SPIRE ${ }^{\mathrm{b}}$ & $500 \mu \mathrm{m}$ & 185 & 36 & $2011 \mathrm{Jul} 12$ & $11.34 \pm 0.15$ \\
\hline
\end{tabular}

Notes. Columns 1: telescope and instrument; 2: filter or bandpass; 3: integration time in seconds; 4: point-spread function, quoted as the seeing disk full width at half-maximum (FWHM) for ground-based instruments, and the tabulated point-spread function FWHM for space observatories; (5) universal date of observation; (6) AB magnitude for all images of the source in aggregate- - see the main text for further details.

a Spitzer data are from Program 70154, "Mass across the Redshift Desert: Stellar Masses in a Large and Uniform Sample of Strongly-Lensed Galaxies at $1<z<3$ " (PI: Gladders).

${ }^{\mathrm{b}}$ Herschel images are from program DDT_jrigby_2, "The far-IR SED of a highly magnified red galaxy” (PI: Rigby).

${ }^{\mathrm{c}}$ Central galaxy flux subtracted using SED model, as detailed in the main text.

observing to within $0.015 \mathrm{mag}$; we thus consider that the $Y$-band data have calibration uncertainties of a few percent. In all four bands, these uncertainties are sub-dominant to other sources of error.

Optical imaging in griz was obtained with the GMOS imaging spectrograph on the Gemini North $8.2 \mathrm{~m}$ telescope; zero points and color terms were established by comparison to SDSSmeasured stars in the same field and are uncertain by a few hundredths of a magnitude.

The GMOS/Gemini-N and FourStar/Magellan imagery is shown in Figure 3, with images centered on the BCG. Table 1 summarizes the details of the observations and data. Photometry 
of the lensed source is reported in Table 1, with details of the measurement process including treatment of the central galaxy given in Section 3.4.

\subsection{WISE Photometry}

SGAS 1438 was included in the Wide-Field Infrared Survey Explorer (WISE) preliminary release (Wright et al. 2010). As it is extended in the WISE bands, we perform aperture photometry rather than take the profile-fit photometry from the WISE preliminary release source catalog. Though the source is detected in the bluest two bands of WISE, these data are completely superseded by the higher spatial resolution and deeper imaging from Spitzer-IRAC, so we report here only the WISE measurements at $12 \mu \mathrm{m}$ and $22 \mu \mathrm{m}$. These wavelengths are uniquely covered by WISE for this source and are important since they measure the strength of mid-IR aromatic emission features. The details of these data and measured photometry are reported in Table 1; details of corrections for contamination by light from the BCG are given in Section 3.4.

\subsection{Herschel Photometry}

We obtained mid- and far-IR photometry for SGAS 1438 in discretionary time on Herschel using the PACS and SPIRE instruments, with a total clock time of $2.1 \mathrm{hr}$. Details of the observations are given in Table 1 .

For PACS, we performed aperture photometry on the level 2 pipleline-produced science images, using annular apertures with radii of 10,12 , and 16 pixels at $70 \mu \mathrm{m}, 12,14$, and 16 pixels at $100 \mu \mathrm{m}$, and 10,12, and 16 pixels at $160 \mu \mathrm{m}$, respectively. We applied aperture corrections as given in technical memo PICC-ME-TN-037 v1.0. We applied color corrections as given in technical memo PICC-ME-TN-038 v1.0 by interpolating to a $27 \mathrm{~K}$ blackbody from the blackbodies tabulated at 20 and $30 \mathrm{~K}$. $^{11}$ These color corrections were 1.09, 1.00, and 0.97 at 70, 100, and $160 \mu \mathrm{m}$. At $70 \mu \mathrm{m}$, where the spectral slope is particularly steep, the uncertainty on the color correction is $\sim 10 \%$, and thus contributes significantly to the photometric uncertainty.

For SPIRE, we performed aperture photometry on the level 2 science images, using annular apertures, following the procedure of the SPIRE Photometry Cookbook. In the Rayleigh-Jeans limit of $F_{v} \propto v^{2}$, the color corrections tabulated in the Cookbook are $0.94,0.95$, and 0.94 at 250, 350, and $500 \mu \mathrm{m}$. Resulting PACS and SPIRE AB magnitudes are listed in Table 1, converted from measured flux densities $f_{v}$ to $m_{A B}=8.9-2.5 \log \left(f_{v}\right)$, with $f_{v}$ in Janskys following the definition of $\mathrm{AB}$ magnitudes (Oke 1974).

SGAS 1438 is bright enough in PACS and SPIRE that the photometric uncertainty is dominated not by photon noise, but by a number of systematic effects: uncertainty in the color correction, aperture correction, and centroiding, and the fact that the source is somewhat resolved in the shortest-wavelength bands. We estimate these effects as contributing a total of $13 \%$ uncertainty to the flux density in each band.

At its band with highest spatial resolution, $70 \mu \mathrm{m}$, Herschel partially resolves SGAS 1438, in that it splits the southern image from the blended other images. By fitting with two GALFIT components (Peng et al. 2010; see Section 3.4), we find that the southern image contains $22 \% \pm 5 \%$ of the $70 \mu \mathrm{m}$ light.

\footnotetext{
11 The PACS photometry is quite sensitive to the choice of color correction; we determined by fitting the photometry that a $T=27 \mathrm{~K}$ blackbody was an adequate fit to the raw (not de-redshifted) photometry. We experimented with modified blackbodies, where $F_{v}^{\text {modified }}=v^{\beta} B_{v}(T)$, but found that these did not provide a better fit.
}

\subsection{Subtracting the Central Galaxy and Final Photometry}

The field of SDSSJ $1438+1454$ is complex: the multiple images of the lensed source encircle the BCG, which contributes significantly to the flux in the optical, near-infrared, and Spitzer 3.6 and $4.5 \mu \mathrm{m}$ bands, and possibly the WISE $12 \mu \mathrm{m}$ and $22 \mu \mathrm{m}$ bands as well. This complexity requires an atypically involved analysis to isolate the flux contribution of the source at each wavelength. To do this, we fit the BCG in each of the optical and near-IR images using GALFIT (Peng et al. 2010). The appropriate point-spread function (PSF) model for each image was constructed from fitting point sources in that image with a set of PSF objects fitted using a multicomponent Moffat+Gaussian model in GALFIT, and then scaled and summed at a fiducial center to produce the final PSF model for each image. For the BCG fits, it was found that a multicomponent Sérsic model-with a small central component that is rounder and about 3-4 mag fainter than the dominant component-was needed to achieve an acceptable fit. That the $\mathrm{BCG}$ requires more than one component for an acceptable fit is neither uncommon nor unexpected, and we have confirmed that this result is not due to a misconstruction of the PSF reference. The lensed source was masked iteratively, with components revealed after an initial BCG subtraction remasked and the fit iterated across all bands with the same mask. Figure 4 shows the final BCG-subtracted images for a few representative wavelengths.

The resulting BCG models do not show any significant evidence for color gradients in the BCG, and so to subtract the BCG flux from the Spitzer $3.6 \mu \mathrm{m}$ and $4.5 \mu \mathrm{m}$ images, we took the best-fit $K_{s}$-band BCG model, convolved it with a PSF constructed as above for the IRAC images, and then subtracted a scaled version of the resulting BCG image by eye. We did not use GALFIT to perform the subtraction automatically because the Spitzer-IRAC PSF badly blends the lens with some of the images of the source, and thus the minimization calculation in GALFIT performs poorly absent more extensive masking of the lensed source.

For the IRAC data, and all images at shorter wavelengths, we then measured the magnitude of the isolated southern image of the source using the techniques described in Wuyts et al. (2010). We also measured the ratio of the total flux of the source to this southern image in the four reddest bands, where the flux ratio of SGAS 1438 to the BCG is highest. This ratio is $3.49 \pm 0.17$. Final magnitudes reported in Table 1 are constructed from the measured magnitudes of the isolated southern image rescaled to the total image using this ratio. The simpler approach of reporting total source magnitudes in the BCG-subtracted images was avoided due to the possible influence of systematics from the BCG model subtraction. At bluer wavelengths, the $\mathrm{BCG}$ is $4-5$ mag brighter than the source, such that aperture measurements of the entire source would be completely dominated by the BCG; by scaling from measurements of the isolated southern image, we trade these potential systematics, which are difficult to quantify, for a noisier measurement and a quantified systematic uncertainty with a scaling of less than 5\%. Note that we do not include this systematic 5\% uncertainty in Table 1; it is only relevant when comparing results at shorter wavelengths to data from Herschel and WISE, and since the spectral energy distribution (SED) fitting (see Section 4.4) is done independently for the shorter and longer wavelengths, it has no effect on our conclusions.

At the long wavelengths sampled by Herschel, the contribution of the BCG is expected to be insignificant. In the WISE 


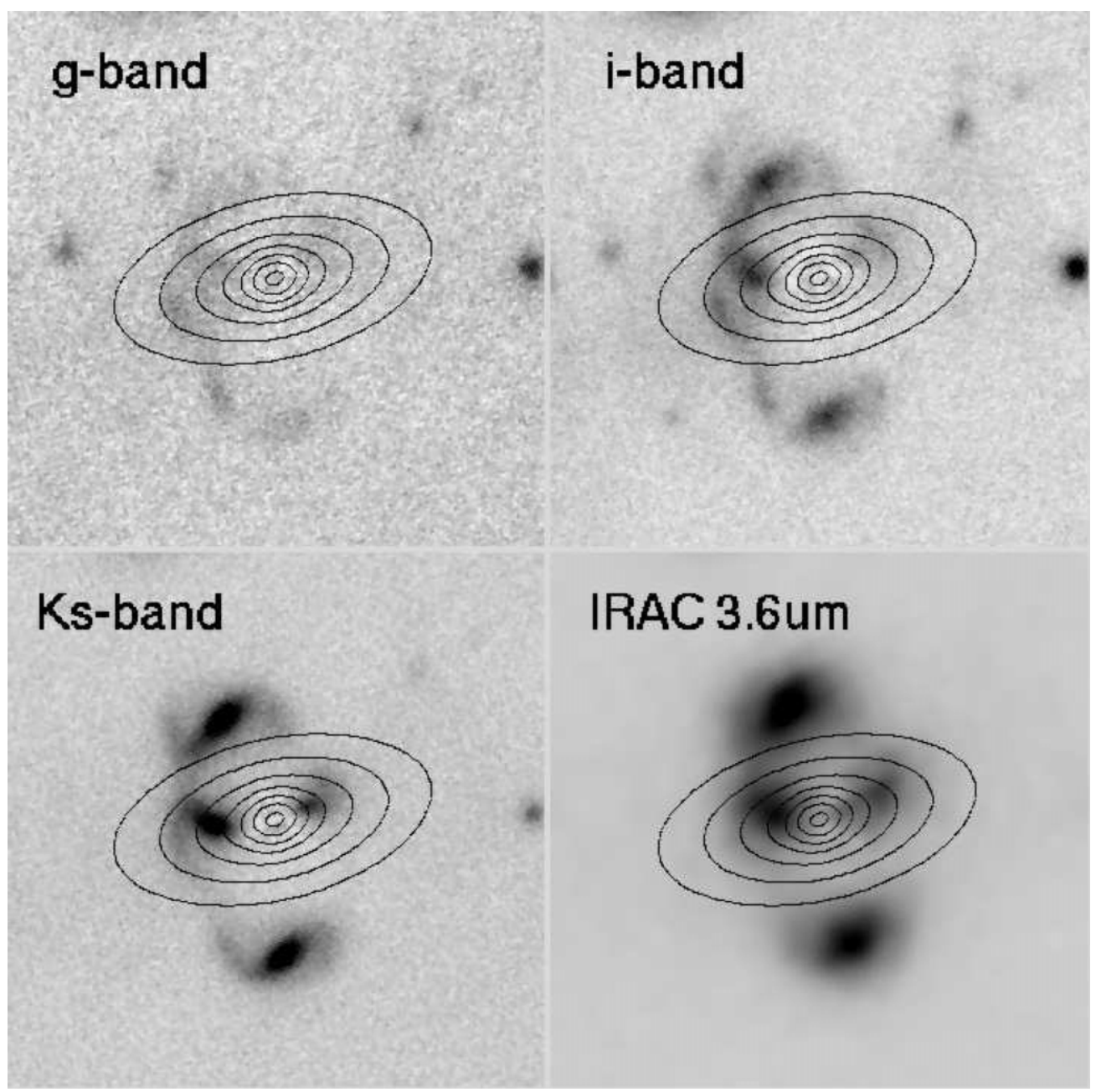

Figure 4. Example images $\left(20 \times 20^{\prime \prime}\right)$ after subtraction of the brightest cluster galaxy (BCG). Residuals near the center of the field are modest, with amplitudes that are $1 \%-2 \%$ of the BCG core prior to subtraction. The BCG location and orientation is indicated by contours taken from the $K_{s}$-band model.

$12 \mu \mathrm{m}$ and $22 \mu \mathrm{m}$ bands, the contribution from the central lens galaxy may be significant, and the poor spatial resolution of WISE does not separate the BCG from the lensed source. We thus proceed by using an elliptical galaxy SED model, anchored at the reddest wavelengths at which we have sufficient resolution to measure the central galaxy light independently $(H K$ and 3.6, $4.5 \mu \mathrm{m})$. We take as the fiducial SED the Spitzer data on BCGs in clusters from Egami et al. (2006). We use the data on central galaxies from the galaxy clusters A773, A2219, and A2261, all of which are close to SDSSJ 1438+1454 in redshift, and are noted in Egami et al. as normal central galaxies with no excess flux at long wavelengths. From these three galaxies, and their $K_{s}$ magnitudes in the 2MASS catalog (Skrutskie et al. 2006), we compute colors for the $4.5 \mu \mathrm{m}, 8 \mu \mathrm{m}$, and $24 \mu \mathrm{m}$ bands referenced to the $K_{s}$ and $3.6 \mu \mathrm{m}$ bands. Uncertainties are taken as the spread in these values across the three galaxies. We measure the $K_{s}$ and $3.6 \mu \mathrm{m} \mathrm{AB}$ magnitudes of the best-fitting BCG model from our data, and then use the colors derived from the data of Egami et al., with simple interpolation, to predict the $12 \mu \mathrm{m}$ and $22 \mu \mathrm{m}$ magnitudes of the central galaxy in SDSSJ $1438+1454$. Final uncertainties are derived from the uncertainty in the color, added in quadrature to the difference between the predicted magnitudes in the $K_{s}$ and $3.6 \mu \mathrm{m}$ bands. The result is that at $12 \mu \mathrm{m}$, the WISE photometry of the lensed source is nominally contaminated at $7 \% \pm 4 \%$ by the central galaxy, and at $22 \mu \mathrm{m}$ at $2.5 \% \pm 1.5 \%$. In both cases, the uncertainty on this correction is less than the photon noise in the measurements.

\subsection{Optical Spectroscopy}

The BCG was observed spectroscopically using the ARC $3.5 \mathrm{~m}$ telescope and the Double Imaging Spectrograph (DIS) on the night of 2011 April 8, to measure its velocity dispersion. The 1".5 slit was used, with the B1200 and R1200 gratings used on the blue and red channels, respectively. The gratings were centered at $4750 \AA$ and $6200 \AA$, which provided coverage from $3870 \AA$ to $5130 \AA$ and $6250 \AA$ to $7390 \AA$ and nominal resolutions of $R \sim 2000$ and $\sim 3000$ at the wavelength centers of the blue and red channels. Three $1800 \mathrm{~s}$ integrations were acquired under good conditions. Data were reduced to flux- and wavelength-calibrated stacked one-dimensional spectra using custom IDL scripts incorporating procedures from the XIDL ${ }^{12}$ software package. The resulting spectra have a per-pixel signalto-noise ratio $(\mathrm{S} / \mathrm{N})$ of typically $\sim 10$ and $\sim 20$ in the blue and red channels, respectively.

\subsection{Near-infrared Spectroscopy}

We obtained a spectrum of SGAS 1438 with the NIRSPEC instrument on the Keck II telescope on the night of UT 2011

\footnotetext{
12 http://www.ucolick.org/ xavier/IDL/index.html
} 


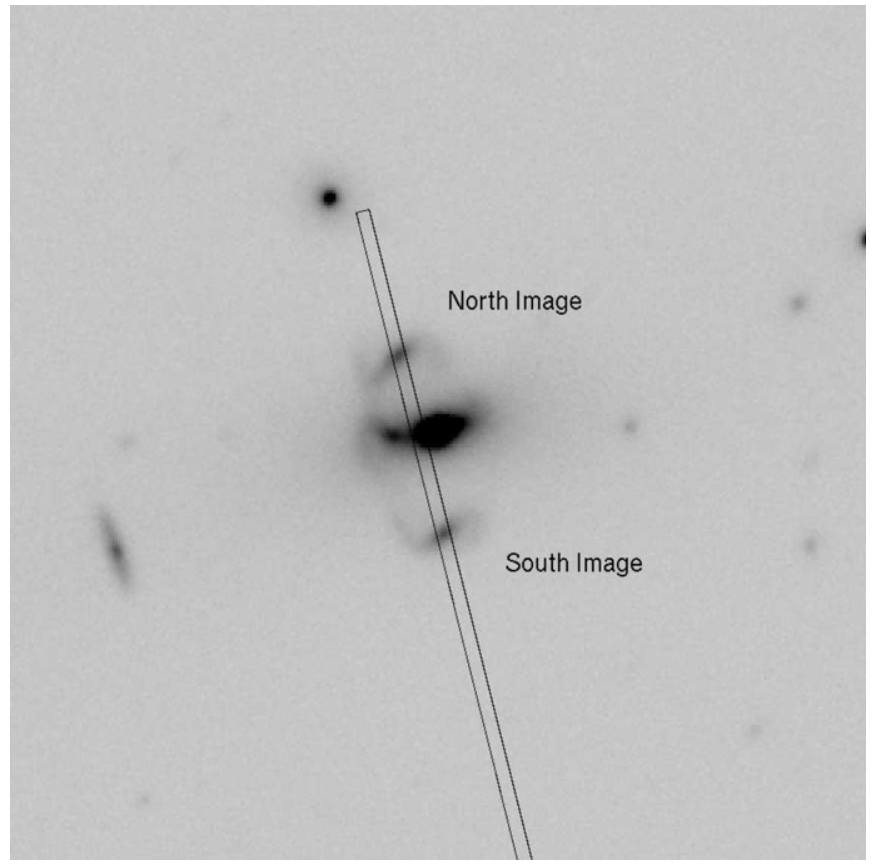

Figure 5. $45 \times 45^{\prime \prime} K_{s}$-band image showing how the Keck NIRSPEC slit was placed to cover two images of the lensed source. The slit is placed at one of the two nod positions.

March 19. The weather was clear, with the seeing variable during the night and especially poor while SGAS 1438 was observed. The airmass was $\sec z<1.07$. The slit position angle was $346^{\circ} \mathrm{E}$ of $\mathrm{N}$, such that spectra for the two brightest images of SGAS 1438 were simultaneously captured, as shown in Figure 5. Some light from the BCG is included in the slit though it is spatially distinct from the light from SGAS 1438. We used the NIRSPEC-2 filter, low-resolution mode, a grating angle of 36.48 , the $0.76 \times 42^{\prime \prime}$ slit, and an ABBA nod pattern with a $14^{\prime \prime}$ nod. This setup covered the wavelength range of $1.11-1.29 \mu \mathrm{m}$. We integrated for $600 \mathrm{~s}$ at each nod position. For telluric flux calibration, the star A0 V star HD 131951 was observed immediately after the observations, at an airmass of $\sec z=1.06$ and a distance of $4^{\circ}$ from SGAS 1438 .

We reduced the NIRSPEC spectra using George Becker's pipeline as described in Rigby et al. (2011), with one exception: since the emission lines were too faint to extract a onedimensional spectrum from each nod, we instead summed the sky-subtracted two-dimensional frames taken in Nod A, repeated the summation for Nod B, and then separately extracted the spectra from each summed nod. For the North image, we weighted-average combined the extracted spectra for each of the two nods. For the South image, we used only the spectrum from Nod B, as the spectrum from Nod A suffered persistence from the trace of a telluric star. The effective integration time was thus 80 minutes for the North image and 40 minutes for the South image.

\section{RESULTS}

\subsection{Velocity Dispersion of the BCG}

The BCG velocity dispersion $\left(\sigma_{v}\right)$ was obtained using the method of Kelson et al. (2000; see Equation (9) therein). Here, redshift and $\sigma_{v}$ are jointly determined by minimizing $\chi^{2}$ as the galactic spectrum is compared to a shifted, broadened, continuum-matched stellar template in pixel space using a gradient search algorithm (e.g., IDL MPFIT). As the $\mathrm{S} / \mathrm{N}$ of the blue channel BCG data was low, we excluded it from this process, using it only to provide initial redshift information based on $\mathrm{Ca} \mathrm{H} \& \mathrm{~K}$. Using both the blue and red channel spectra in their entirety, we find a redshift for the BCG of $z_{\mathrm{BCG}}=0.2373 \pm 0.0002$. Unfortunately, the loss of the blue channel data combined with the BCG redshift and the fixed disperser available to remote observers on DIS removed any overlap between BCG and observed stellar standards, rendering the latter unusable as templates. We employed instead a set of high-resolution spectra from the UVES Paranal Observatory Project (Bagnulo et al. 2003), selected by spectral type without regard for metallicity.

Before comparison with the BCG spectrum, it was necessary to match the template resolution with that of DIS. The latter quantity was determined in two ways: (1) Gaussian fitting of sky emission lines on 2D DIS spectra and (2) Gaussian broadening of UVES templates and comparison to DIS stellar spectra. The methods yielded values of FWHM $_{\text {inst }}=1.46 \pm 0.15 \AA$ and $1.78 \pm 0.21 \AA$, respectively, showing no significant trend with wavelength. We adopted the average of these, $\mathrm{FWHM}_{\text {inst }}=$ $1.62 \AA$, as the instrumental resolution for DIS, corresponding to $\sigma_{\text {inst }} \sim 30 \mathrm{~km} \mathrm{~s}^{-1}$ at the relevant wavelength and observed frame. All templates were broadened to this resolution before beginning the BCG fitting procedure.

At the $\mathrm{S} / \mathrm{N}$ of our BCG spectrum, the uncertainties associated with template variation/mismatching (systematic) and photon statistics (random) are dominant and of a comparable order. Precisely quantifying the systematic effects requires extensive simulation and is necessary when investigating detailed physical relations based on velocity dispersions, such as $M_{b}-\sigma$. Our interest in this quantity, however, extends only to providing a prior for the lensing model below. Hence, we deem it sufficient to verify that our procedure adequately reproduces accepted values of $\sigma_{v}$ from the literature. We took as a test sample a set of spectra for 20 Virgo ellipticals from Dressler (1984) for which $\sigma_{v}$ measurements were made using a region surrounding the $\lambda 5176 \mathrm{Mg} b$ triplet—which we also capture. The Dressler (1984) data are of comparable $\mathrm{S} / \mathrm{N}$ to our own. Restricting the fitting to the 4900-5400 $\AA$ interval used in the original paper, the template that best-reduced $\chi^{2}$ across all of the (Dressler 1984) galaxies yielded $-0.02<\log _{10}\left(\sigma_{v} / \sigma_{v, D 84}\right)<0.05$ for $\sigma_{v, D 84} \geqslant$ $85 \mathrm{~km} \mathrm{~s}^{-1}$, with a difference in the mean consistent with zero. Template-to-template variations introduce a logarithmic scatter of less than 0.1 dex across all $\sigma_{v}$, but do not bias the results.

By adopting as much of the Dressler (1984) interval as our data allow $(\lambda \in[5024,5400] \AA)$ and masking telluric features, we find $\sigma_{v}$, BCG $=298 \pm 8$ (random) \pm 18 (systematic) $\mathrm{km} \mathrm{s}^{-1}$. Values are taken from the best-fitting template with the systematic error reflecting rms variation across templates.

\subsection{Lensing Model and Magnification}

We construct a lens model of the cluster using the publicly available software LENSTOOL (Jullo et al. 2007). The cluster and the BCG are each modeled by a pseudo-isothermal ellipsoid mass distribution (PIEMD; Limousin et al. 2005), ${ }^{13}$ parameterized by its position $x, y$; a fiducial velocity dispersion $\sigma_{\text {PIEMD }}$; a core radius $r_{\text {core }}$; a cut radius $r_{\text {cut }}$; ellipticity $e=\left(a^{2}-b^{2}\right) /\left(a^{2}+b^{2}\right)$, where $a$ and $b$ are the semi-major and semi-minor axes, respectively; and a position angle $\theta$. We fix 13 This profile is formally the same as a dual pseudo isothermal elliptical mass
distribution (dPIE; see Elíasdóttir et al. 2007). 


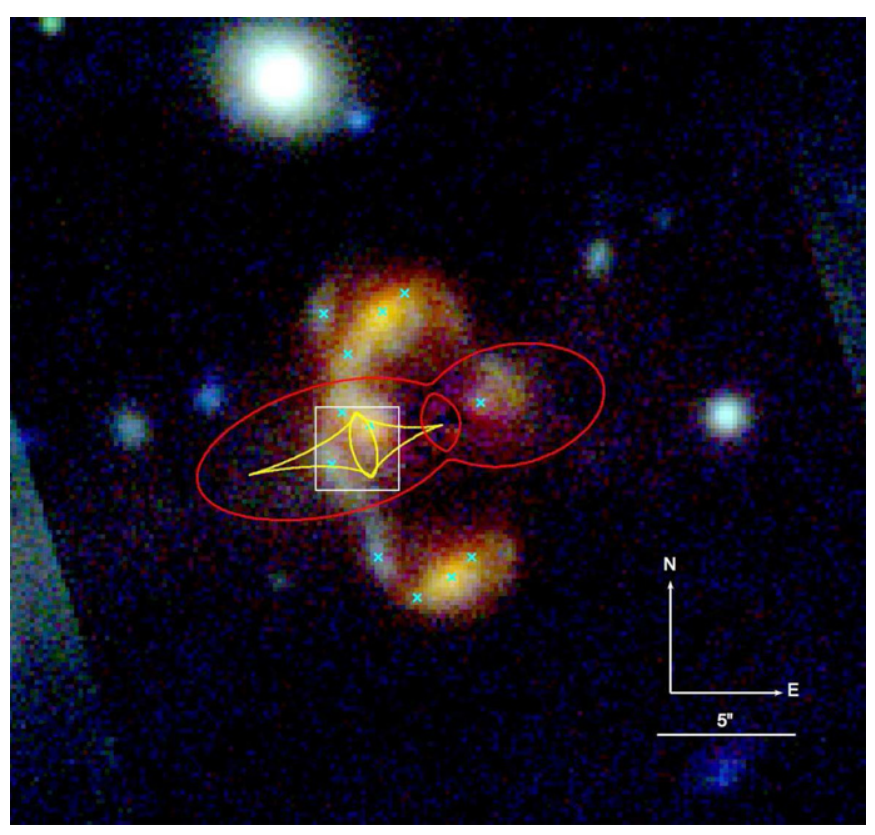

Figure 6. Lens model: critical curves (red lines) and caustics (yellow lines) from the best-fit lens model are overplotted on a color composite image (red: $J$, green: $i$, blue: $g$ ). The coordinates within each arc that were used as lensing constraints are marked in cyan. The location of the source in the source plane (see Figure 7) is indicated by a white box.

(A color version of this figure is available in the online journal.)

the BCG parameters $x, y, e$, and $\theta$ at their observed values as derived from the GALFIT modeling described above and fix the cluster cut radius at $1000 \mathrm{kpc}$ as this cannot be constrained by the data. This does not significantly affect the lensing model. The BCG velocity dispersion of $\sigma_{v}=298 \pm 26 \mathrm{~km} \mathrm{~s}^{-1}$ measured in Section 4.1 is set as a Gaussian prior. The velocity dispersion of the cluster was not measured, but we can estimate it. SDSSJ 1438+1454 appears in the GMBCG catalog (Hao et al. 2010) with a weighted richness $N_{\text {gals }}^{\text {weighted }}=9.734$; following the calibration of Becker et al. (2007), we estimate a velocity dispersion of $\sigma=318 \pm 111 \mathrm{~km} \mathrm{~s}^{-1}$. The low richness and velocity dispersion indicate that SDSSJ $1438+1454$ is a poor cluster or group, in agreement with the general appearance of the cluster in the optical imaging, and the small separation between the lensed images of the background galaxy. We use this velocity dispersion as a Gaussian prior. Note that the above velocity dispersions are converted to $\sigma_{\text {PIEMD }}$ using the relations in Elíasdóttir et al. (2007). We also note that the BCG in this cluster is very dominant, based on the magnitude difference of 1.64 mag in the $i$ band between the BCG and the next two brightest cluster members (for typical poor clusters, this difference is $<1.0 \mathrm{mag}$; Niederste-Ostholt et al. 2010). We therefore limit the cluster center to be no farther than $39^{\prime \prime}$ from the BCG, which is the separation between the BCG and the next brightest galaxy. Dominant systems tend to show good alignment between the BCG and the cluster halo (Niederste-Ostholt et al. 2010), similar to the results from the lens modeling shown below. The best-fit model is found via Monte Carlo Markov Chain (MCMC) optimization in the image plane, using one to four coordinates in each instance of the lensed galaxy as constraints (see Figure 6). The best-fit model has an image-plane rms of 0 '. 1 . Table 2 lists the best-fit parameters and their $1 \sigma$ uncertainties.

Since the lensed images are extended, the magnification is not fixed across different portions of each lensed image of the source. The average magnification of each lensed image is thus
Table 2

Best-fit Lens Model Parameters

\begin{tabular}{lccccccc}
\hline \hline $\begin{array}{l}\text { Halo } \\
\text { (PIEMD) }\end{array}$ & $\begin{array}{c}\text { R.A. } \\
\left({ }^{\prime \prime}\right)\end{array}$ & $\begin{array}{c}\text { Decl. } \\
\left({ }^{\prime \prime}\right)\end{array}$ & $e$ & $\begin{array}{c}\theta \\
(\mathrm{deg})\end{array}$ & $\begin{array}{c}r_{\text {core }} \\
(\mathrm{kpc})\end{array}$ & $\begin{array}{c}r_{\text {cut }} \\
(\mathrm{kpc})\end{array}$ & $\begin{array}{c}\sigma_{\text {PIEMD }} \\
\left(\mathrm{km} \mathrm{s}^{-1}\right)\end{array}$ \\
\hline Cluster & $7.7_{-4.9}^{+2.3}$ & $-2.2_{-0.8}^{+1.6}$ & $0.54_{-0.30}^{+0.06}$ & $165_{-4}^{+4}$ & $12_{-6}^{+1}$ & {$[1000]$} & $672_{-222}^{+50}$ \\
BCG & {$[0]$} & {$[0]$} & {$[0.64]$} & {$[167]$} & $1.3_{-1.0}^{+2.0}$ & $57_{-46}^{+23}$ & $267_{-30}^{+71}$ \\
\hline
\end{tabular}

Notes. All coordinates are measured in arcseconds relative to the center of the BCG, at [R.A., decl.] $=[219.68768,14.903507]$. The ellipticity is expressed as $e=\left(a^{2}-b^{2}\right) /\left(a^{2}+b^{2}\right) . \theta$ is measured north of west. Error bars correspond to the $1 \sigma$ confidence level as inferred from the MCMC optimization. Values in square brackets are for parameters that are not optimized.

Table 3

Magnifications of Images of SGAS 143845.1+145407

\begin{tabular}{lcl}
\hline \hline Image & Magnification & \multicolumn{1}{c}{ Notes } \\
\hline North & $6.5_{-2.5}^{+1.8}$ & Complete image \\
South & $5.2_{-2.1}^{+1.6}$ & Complete image \\
East & $2.9_{-0.8}^{+1.4}$ & Partial image \\
West & $4.0_{-1.3}^{+2.5}$ & Partial image \\
North core & $4.6_{-1.8}^{+1.7}$ & NIR slit region \\
South core & $5.8_{-2.2}^{+2.1}$ & NIR slit region \\
All & $16.8_{-6.2}^{+5.2}$ & \\
\hline
\end{tabular}

Notes. Columns 1: a source image name-see Figure 6; 2: the estimated magnification from the strong lensing model, and 3: notes about the nature of each image. Due to the lensing configuration, two images are complete images of the source, and the other two are partial images only. The magnification of the "All" image gives the magnification of all four images in aggregate relative to a single unlensed instance of the source; this is the appropriate magnification to use when converting the observed photometry in Table 1 back to unlensed quantities.

measured as the ratio of areas occupied by the galaxy in the image plane and source plane. In Table 3, we report the average magnification for each image of the source. We also report the magnification of the core regions of the North and South images, as these regions were targeted for spectroscopy (see Figure 5). Table 3 also includes the total magnification of the aggregate of all of the images of the source; note that the total magnification of the source is not a linear sum of the magnifications of its instances because the source galaxy crosses the source-plane caustics and some of its lensed instances (the east and west images) represent only parts of the source galaxy rather than all of it. In Figure 7, we show the magnification contours of the best-fit model and a source-plane reconstruction of the galaxy. To estimate the uncertainty on each magnification, we draw parameters from chains in the MCMC process, compute a lens model for each set of parameters, and measure the magnification of each image. The uncertainties quoted in Table 3 are given as the range of magnifications corresponding to $1 \sigma$ in parameter space.

\subsection{Redshift, Line Fluxes, and Metallicity}

We separately fit the Keck NIRSPEC spectra for each of the two images of the lensed galaxy, as follows. The redshift was set initially by fitting a single Gaussian to $\mathrm{H} \alpha$. We then simultaneously fit the [N II] 6549.85, 6585.28 $\AA$ doublet and the $6564.61 \AA \mathrm{H} \alpha$ line,${ }^{14}$ using three Gaussians using the IDL Levenberg-Marquardt least-squares fitting code MPFITFUN

14 Wavelengths are vacuum, from NIST at 

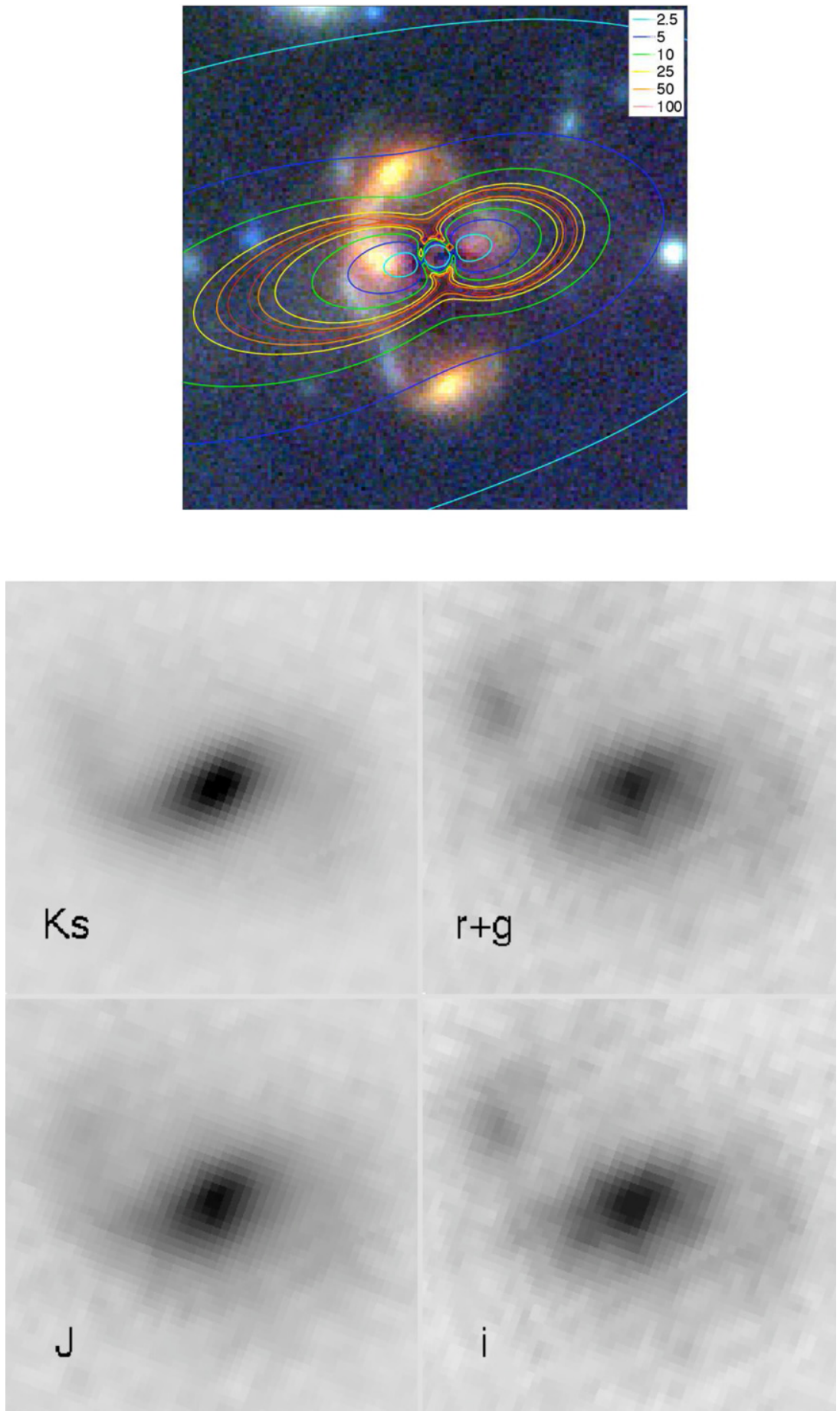

Figure 7. Lens model: left: contours of absolute magnification are overplotted on a color composite image (red: $J$, green: $i$, blue: $g$ ) of SGAS 1438 . Right: reconstruction of the source of SGAS 1438, from the best-fit lens model. We ray-trace each pixel in the image plane through the lens model and resample the source plane into a 0.05 pixel grid.

(A color version of this figure is available in the online journal.)

(Markwardt 2009). Wavelengths were set at the NIST values ${ }^{15}$ and allowed to vary within $\pm 2 \sigma$ of the initial fit to $\mathrm{H} \alpha$. A common linewidth for all of the lines was allowed to vary freely. The [N II] doublet flux ratio was fixed at the value computed in Storey \& Zeippen (2000).

Table 4 reports the redshift for SGAS 1438 as measured from simultaneously fitting the $[\mathrm{N}$ II] and $\mathrm{H} \alpha$ lines in each near-IR spectrum. The result is consistent with that found by fitting

\footnotetext{
15 http://www/pa.uky.edu/ peter/atomic
}

$\mathrm{H} \alpha$ alone. The weighted mean redshift for the two images is $z=0.81589 \pm 0.00005$. The two images have identical redshifts within uncertainties. The spectra are plotted, with their multicomponent fits, in Figure 8. Table 4 lists measured fluxes for $\mathrm{H} \alpha$ and $[\mathrm{N}$ II].

We infer metallicities from the measured $\mathrm{H} \alpha$ to $\left[\mathrm{N}_{\mathrm{II}}\right]$ line flux ratios using the calibration of Pettini \& Pagel (2004). The metallicity is calculated for each image of the lensed galaxy and tabulated in Table 4. The summed [N $\mathrm{NI}_{\mathrm{II}}$ and $\mathrm{H} \alpha$ fluxes over both images yield the most precise metallicity: 
Table 4

Measured Emission Lines in SGAS 143845.1+145407

\begin{tabular}{|c|c|c|c|c|c|c|}
\hline Object & Redshift & $f(\mathrm{H} \alpha)$ & $f\left(\mathrm{~N}_{\text {II }} 6585\right)$ & {$[\mathrm{N}$ II $] / \mathrm{H}-\alpha$} & $12+\log (\mathrm{O} / \mathrm{H})$ & $12+\log (\mathrm{O} / \mathrm{H})$ \\
\hline North image & $0.81588 \pm 0.00006$ & $229 \pm 16$ & $83 \pm 14$ & $0.36 \pm 0.07$ & $8.65_{-0.05}^{+0.04}$ & $8.69_{-0.09}^{+0.08}$ \\
\hline South image & $0.8159 \pm 0.0001$ & $238 \pm 28$ & $69 \pm 20$ & $0.29 \pm 0.12$ & $8.59_{-0.09}^{+0.07}$ & $8.59_{-0.14}^{+0.12}$ \\
\hline both images & $0.81589 \pm 0.00005$ & $467 \pm 32$ & $152 \pm 24$ & $0.33 \pm 0.07$ & $8.62_{-0.06}^{+0.05}$ & $8.64_{-0.09}^{+0.09}$ \\
\hline
\end{tabular}

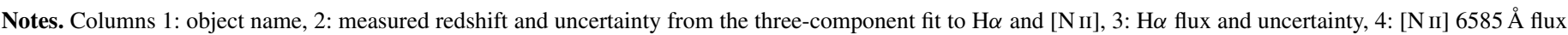

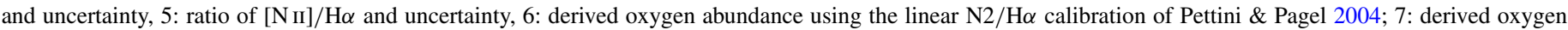

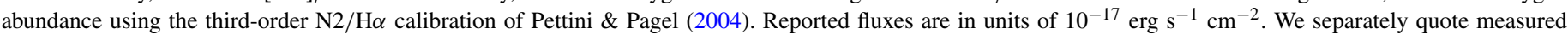

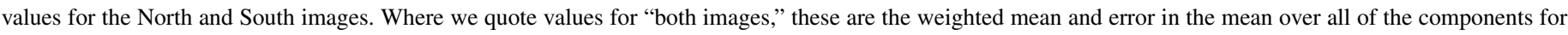
the redshift, the sum over all of the components for each flux, and the ratio of the summed fluxes for the line ratio.
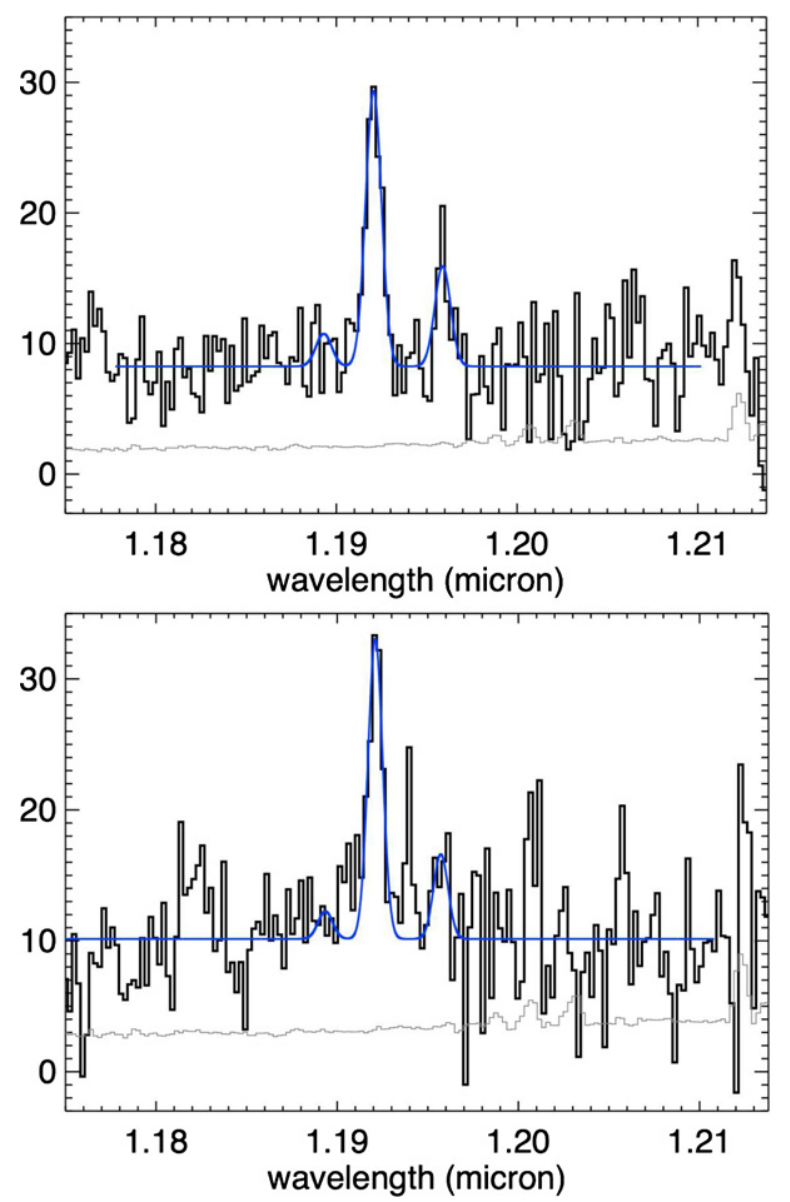

Figure 8. Observed Keck/NIRSPEC spectra of $\mathrm{H} \alpha$ and [N II] in SGAS 1438. Spectra of the North and South images are plotted in the left and right panels, respectively. Each $X$-axis shows observed wavelength in $\mu \mathrm{m}$, and each $Y$-axis shows observed specific intensity $f_{\lambda}$ in units of $10^{-17} \mathrm{erg} \mathrm{s}^{-1} \mathrm{~cm}^{-2} \AA^{-1}$. In each panel, the spectrum is plotted in black, the $1 \sigma$ uncertainty spectrum is plotted in gray, and the multi-component Gaussian fit is plotted in blue. The extent of the blue line shows the region over which the continuum was fitted. In each spectrum, the three fitted emission lines are [N II] 6548, H $\alpha$, [N II] 6583.

(A color version of this figure is available in the online journal.)

$12+\log (\mathrm{O} / \mathrm{H})=8.62 \pm 0.06$ using the linear Pettini \& Pagel calibration, and $12+\log (\mathrm{O} / \mathrm{H})=8.64 \pm 0.09$ using their thirdorder polynomial calibration. For reference, solar metallicity is $12+\log (\mathrm{O} / \mathrm{H})=8.69 \pm 0.05$ (Asplund et al. 2009). Thus, we conclude that SGAS 1438 has solar metallicity.

\subsection{Spectral Energy Distribution Fits}

We fit the rest-frame UV, optical, and near-infrared SED of SGAS 1438 following the procedure outlined in Wuyts

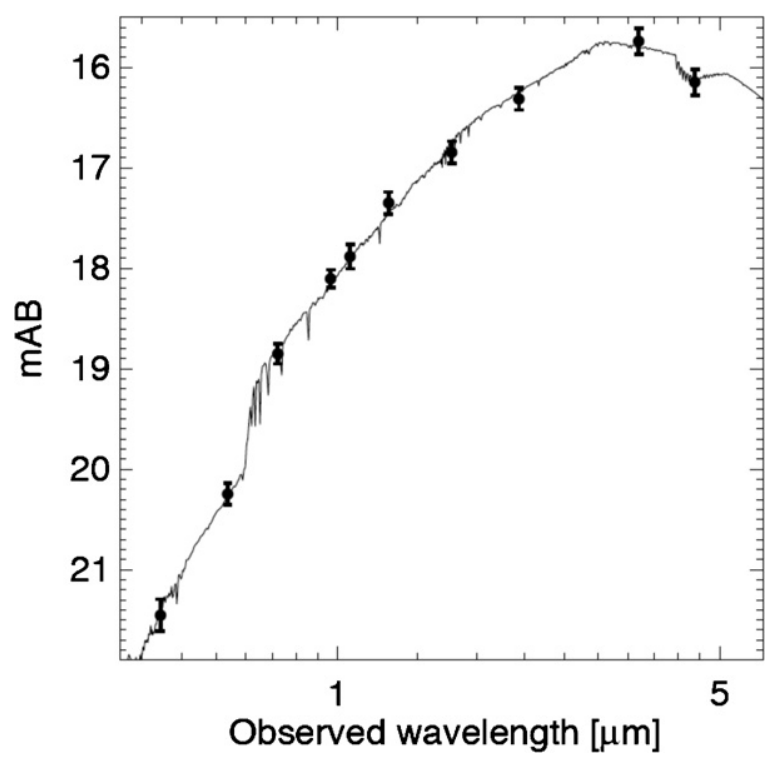

Figure 9. Photospheric spectral energy distribution of the lensed source SGAS 1438. Datapoints correspond to gri photometry from Gemini, $Y J H K_{s}$ photometry from Magellan, and 3.6-4.5 $\mu \mathrm{m}$ photometry from Spitzer, as detailed in Table 1 and described in Section 3. Also plotted is the bestfitting Bruzual \& Charlot stellar population synthesis model, as described in Section 4.4. The units of the $Y$-axis are AB magnitudes. Photometry has been summed over all of the images of SGAS 1438.

et al. (2010, 2012). We use Hyperz (Bolzonella et al. 2000) to perform SED fitting at fixed spectroscopic redshift with the newest Bruzual \& Charlot population synthesis models (CB07; see Bruzual \& Charlot 2003), using the Chabrier initial mass function (Chabrier 2003) and the Calzetti dust extinction law (Calzetti et al. 2000). We allow a range of exponentially declining star formation histories with $e$-folding times $\tau$ ranging from $10 \mathrm{Myr}$ to $2 \mathrm{Gyr}$ as well as a constant star formation history. The age of the stellar population is allowed to vary between $50 \mathrm{Myr}$ and the age of the universe at $z=0.816 .{ }^{16}$ Informed by the near-IR spectroscopy, we fix the metallicity at the solar value. Figure 9 plots the rest-frame UV to near-IR photometry reported in Table 1 as well as the best-fit stellar population model. From this SED, the derived stellar mass, corrected for lensing, is $\log \left(M_{*} / M_{\odot}\right)=10.8_{-0.2}^{+0.3}$.

The Herschel photometry shows that SGAS 1438 is extremely bright in the far-IR. Its infrared SED peaks in $\log \left(f_{v}\right)$ between 90 and $140 \mu \mathrm{m}$ in the rest frame, which is a considerably redder peak wavelength than nearby template galaxies like M 82 or Arp 220 (which peak in $f_{v}$ at $85 \mu \mathrm{m}$ and $70 \mu \mathrm{m}$, respectively.) We fit the $12-500 \mu \mathrm{m}$ photometry with the local galaxy template set of

\footnotetext{
16 6.556 Gyr in our assumed cosmology.
} 


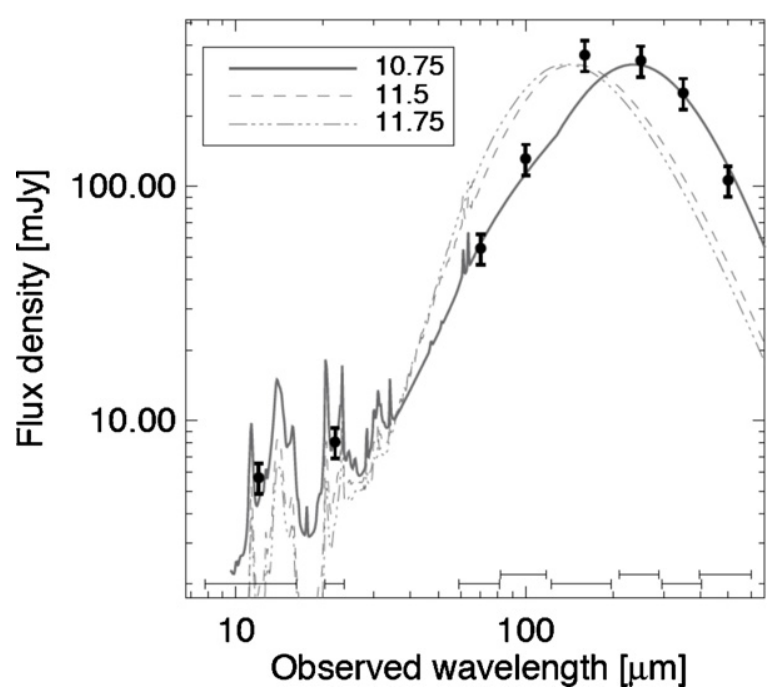

Figure 10. Dust-reprocessed spectral energy distribution of the lensed source SGAS 1438. Datapoints correspond to observed photometry at observed wavelengths of 12 and $22 \mu \mathrm{m}$ from WISE, at 70, 100, and $160 \mu \mathrm{m}$ from Herschel/PACS, and at 250, 350, and $500 \mu \mathrm{m}$ from Herschel/SPIRE, as detailed in Table 1. Wavelength ranges for each observation are plotted toward the bottom. Also plotted is the best-fitting local-galaxy template (solid line) from Rieke et al. (2009), which is the $\log (L(T I R))=10.75 L_{\odot}$ template. We measure the $L$ (TIR) of SGAS 1438 as $7 \times$ higher than this fiducial template; in other words, the template that best fits SGAS 1438 was made from relatively lowluminosity galaxies, and has been scaled up in luminosity by a factor of seven to fit a galaxy with a much higher luminosity. To illustrate this, we plot for comparison the Rieke templates whose luminosities bracket that of the source: $\log (L(\mathrm{TIR}))=11.5$ and $11.75 L_{\odot}$; these have been normalized to the same peak flux density as the best-fitting template. Clearly, these templates are hotter and do not fit the SED shape of SGAS 1438.

Rieke et al. (2009), by convolving each template with the WISE, PACS, and SPIRE filter curves. As shown in Figure 10, the bestfitting template is the $\log (L(T I R))=10.75 L_{\odot}$ Rieke template. ( $L$ (TIR) is defined as the rest-frame $8-1000 \mu \mathrm{m}$ luminosity.) Correcting for lensing magnification ${ }^{17}$ and integrating this bestfitting template from rest-frame $8-1000 \mu \mathrm{m}$ yields an intrinsic far-infrared luminosity of $L(\mathrm{TIR})=4.0_{-1.1}^{+1.8} \times 10^{11} L_{\odot}$. This best-fitting template peaks in $f_{v}$ at a rest-frame wavelength of $130 \mu \mathrm{m}$.

It is important to note that the best-fitting template was made from the photometry of galaxies almost a decade less infrared luminous than SGAS 1438: the template's luminosity is $7 \times$ lower than our measured $L$ (TIR). In other words, templates of comparable luminosity provide poor fits; their SEDs are much hotter, peaking in $f_{v}$ at rest wavelengths of $75-80 \mu \mathrm{m}$. Thus, matched-luminosity templates provide a poor fit to the shape of the far-IR SED. By contrast, a less luminous template with an $f_{v}$ peak of $130 \mu \mathrm{m}$, when scaled up in luminosity, provides a much better fit to the far-IR SED. We will return to this point in Section 5.

\subsection{Star Formation or AGN?}

An important question when deriving physical conditions for SGAS 1438 is whether there is any significant active galactic nucleus (AGN) contribution to the observed emission, either from an AGN in the background lensed galaxy itself, or from an AGN in the central BCG (the foreground lens galaxy). Here, we review the (lack of) evidence for an AGN. The bulk of the

\footnotetext{
17 Note that the two most magnified images in this case (North and South) are complete, and so we do not expect significant differential magnification effects (Hezaveh et al. 2012).
}

light at near-IR wavelengths and blueward clearly comes from an extended source, both for the lensed source itself and the BCG. The DIS optical spectrum of the BCG does not show any emission lines: the observed spectra include the expected wavelength of [O II] 3727. In the NIR spectrum of the lensed source, the $\mathrm{H} \alpha$ line is not unusually broad or strong. Thus, there is no evidence for a significant AGN component in the rest-frame optical or near-IR.

The reasonable match between the FIR SED and the starforming template set of Rieke et al. (2009) also gives some encouragement that any putative AGN contribution is no larger than might be disguised in that template set. The far-IR SED is matched by a composite galaxy template; an AGN would be expected to make hotter dust and thus shift the peak to the blue, which is not observed. We note that the Herschel photometry at $70 \mu \mathrm{m}$ (Section 3.3), which partially resolves the multiple lensed images, also provides a limit on any AGN component from the BCG. The measured flux in the South image of SGAS 1438 is $22 \% \pm 5 \%$ of the total flux. The predicted fraction from the lens model is $31_{-12}^{+20} \%$, and from the measured flux ratios at shorter wavelengths that resolve all components of the source it is $28 \% \pm 2 \%$. Taken at face value, the $70 \mu \mathrm{m}$ and shorter wavelength flux ratios limit the contribution of the BCG at $70 \mu \mathrm{m}$ to less than $21 \%$ of the total flux, and are consistent with no contribution at all. Finally, there is also no evidence for emission at the position of SGAS 1438 in either the FIRST radio data (Becker et al. 1995) or at X-ray wavelengths in the ROSAT All Sky Survey, although these data do not set any strong limits on a low-luminosity AGN contribution. Absent data at any wavelength that suggest the presence of an AGN, we proceed under the assumption that the emission observed from the lensed source is powered by stars only.

\subsection{Star Formation Rate}

We estimate the star formation rate (SFR) of SGAS 1438 from two methods: $\mathrm{H} \alpha$ and the far-infrared.

To convert the $\mathrm{H} \alpha$ fluxes reported in Table 4 into an SFR captured by the NIRSpec slit, we apply the calibration of Kennicutt (1998), modified for a Chabrier (2003) initial mass function:

$$
\mathrm{SFR}=0.66 L(\mathrm{H} \alpha) \times 7.9 \times 10^{-42} .
$$

With $L(\mathrm{H} \alpha)$ specified in erg $\mathrm{s}^{-1}$, this is the apparent SFR in $M_{\odot} \mathrm{yr}^{-1}$ in the slit; it requires an aperture correction, magnification, and extinction correction to get the intrinsic SFR. Poor and variable seeing during the Keck observation means that slit losses are high and uncertain. We estimate the seeing by convolving the $J$-band image of the BCG until it matches the width of the BCG in the two-D spectrum, and then compute the slit losses by examining the seeing-degraded images of the source. We conclude that the aperture correction is large: a factor of $4 \pm 1$. Final intrinsic SFRs from the $\mathrm{H} \alpha$ measurements are computed for each image, combining uncertainties in the line flux (Table 4), the magnification (Table 3), and the aperture correction. We find $\mathrm{SFR}=31_{-11}^{+21} M_{\odot} \mathrm{yr}^{-1}$ from the North image and $\mathrm{SFR}=25_{-9}^{+16} M_{\odot} \mathrm{yr}^{-1}$ from the South image. Note that these are both measurements of the total SFR for the entire source; combining with equal weights yields a final best value of $\mathrm{SFR}_{\mathrm{H}-\alpha}=28_{-7}^{+13} M_{\odot} \mathrm{yr}^{-1}$.

We also estimate the SFR from the far-infrared luminosity, using the template fitting discussed in Section 4.4 and plotted in Figure 10. The integrated rest-frame $8-1000 \mu \mathrm{m}$ luminosity, corrected by the total magnification, is $4.0_{-1.1}^{+1.8} \times 10^{11} L_{\odot}$. 
Table 5

Morphology and Size of SGAS 143845.1+145407, Sérsic, and Gaussian Profile Fits

\begin{tabular}{|c|c|c|c|c|c|c|c|}
\hline \multirow[t]{2}{*}{ Image } & \multirow[t]{2}{*}{ Filter } & \multicolumn{3}{|c|}{ Image Plane } & \multicolumn{3}{|c|}{ Source Plane } \\
\hline & & $R_{e}(\mathrm{kpc})$ & Sérsic Index & Gaussian $\sigma(\mathrm{kpc})$ & $R_{e}(\mathrm{kpc})$ & Sérsic Index & Gaussian $\sigma(\mathrm{kpc})$ \\
\hline \multirow[t]{4}{*}{ South image } & $K_{s}$ & 3.6 & 0.99 & 7.0 & 3.7 & 1.19 & 7.0 \\
\hline & $H$ & 3.6 & 0.96 & 7.2 & 4.6 & 1.50 & 8.2 \\
\hline & $J$ & 4.0 & 0.71 & 8.4 & 4.8 & 1.66 & 8.4 \\
\hline & SDSS- $i$ & 4.3 & 0.74 & 7.7 & 5.1 & 1.22 & 7.0 \\
\hline \multirow[t]{4}{*}{ North image } & $K_{s}$ & 4.0 & 1.01 & 6.9 & 3.9 & 1.18 & 6.1 \\
\hline & $H$ & 4.1 & 1.01 & 7.3 & 3.8 & 1.20 & 6.2 \\
\hline & $J$ & 4.9 & 0.73 & 9.1 & 3.7 & 1.03 & 6.4 \\
\hline & $\mathrm{SDSS}-i$ & 4.6 & 0.78 & 7.8 & 4.5 & 0.87 & 7.2 \\
\hline Average & All & $4.1 \pm 0.6$ & $0.87 \pm 0.14$ & $7.7 \pm 0.8$ & $4.3 \pm 0.6$ & $1.23 \pm 0.25$ & $7.1 \pm 0.9$ \\
\hline
\end{tabular}

Notes. Columns 1: source image name, 2: filter, 3-5: effective radius, Sérsic index, and Gaussian size in the source plane from fits in the image plane, and 6-8: effective radius, Sérsic index, and Gaussian size from fits to the reconstructed source plane images. In the case of the image plane, effective radii and Gaussian sizes are computed in the source plane simply by dividing by the square root of the magnification, as this is the limiting case suggested by the only modest distortions seen in the magnified images in the image plane. No such correction is applied to fits in the source plane, however, the treatment of the PSF is commensurately more complex, as detailed in the main text.

Via the calibration of Kennicutt (1998), this corresponds to an SFR of $41_{-11}^{+18} M_{\odot} \mathrm{yr}^{-1}$.

\subsection{Extinction}

If we attribute to extinction the higher value of the SFR measured from the FIR versus $\mathrm{H} \alpha$, then the extinction at $\mathrm{H} \alpha$ is $0.4_{-0.4}^{+0.7}$ mag. The extinction at $\mathrm{H} \alpha$ estimated from the SED fit is $0.9_{-0.4}^{+0.7}$ mag. Thus, SGAS 1438 may have a slightly higher extinction than the calibrating sample of Kennicutt (1998), though only at $1 \sigma$ significance.

\subsection{Physical Size and Structural Properties}

The good image quality of the ground-based imaging of SGAS 1438 allows a robust measure of the galaxy's size and morphology. The strong lensing model for SGAS 1438 also shows unusually low distortion for the images of this source, compared, for example, to the highly magnified giant arcs that are more typical in strong lensing galaxy clusters (e.g., Sharon et al. 2012); this suggests that measurements in the image plane can be simply converted to the source plane. To thoroughly explore the source morphology and size, we have fit the North and South images of the galaxy both directly in the image plane and also in reconstructed images in the source plane. We consider the $J H K_{s}$ images as well as the $i$-band image; these are the deepest and best-seeing images, spanning two distinct instruments and telescopes. Visual examination of the initial images of SGAS 1438 (see Figure 3) suggests a two-arm spiral morphology for the source. In fitting the source using simple models, we mask the obvious portions of these apparent spiral arms.

To fit in the image plane, we use GALFIT and the PSF references already established for modeling the BCG light, and fit the isolated (and complete) North and South images of SGAS 1438 independently in the BCG-subtracted frames. Fits were made using both a single Sérsic profile, ${ }^{18}$ as well as a single Gaussian profile to facilitate comparison to the measurements of Rujopakarn et al. (2011). Lengthscales are converted to the source plane by dividing by the square root of the magnification; in the case of equal magnification radially and tangentially, as is

\footnotetext{
18 An exponential disk has a Sérsic index of 1, and a de Vaucouleur profile has a Sérsic index of 4.
}

approximately true here, this is appropriate. The results of this fitting are reported in Table 5. Treating each image in each filter with equal weight, the measured Sérsic index in the image plane is $0.87 \pm 0.14$ with an effective radius of $4.1 \pm 0.6 \mathrm{kpc}$ and a Gaussian size (in $\sigma$ ) of $7.7 \pm 0.8 \mathrm{kpc}$. To measure morphology in the source plane, we construct source plane images from the image plane using the lensing model, by simple interpolation on the irregular grid of image pixels produced in the source plane. This is not optimal from a signal-to-noise perspective, since it treats all image plane pixels equally, regardless of magnification; it is, however, sufficient for the purposes of this paper. To obtain a PSF reference, we map the image plane PSFs back to the source plane in the same way, at the position of the center of the source. Formally, in the source, the realized PSF is a function of position; again, because the distortions are not strong, this effect is sub-dominant. We have tested a range of PSFs sampled from locations around the center of the source and find no significant effect on the results. Again treating each image in each filter with equal weight, the measured Sérsic index in the source plane is $1.23 \pm 0.25$, with an effective radius of $4.3 \pm 0.6 \mathrm{kpc}$ and a Gaussian size of $7.1 \pm 0.9 \mathrm{kpc}$.

These measurements, which are consistent across four filters, two images of the source, and both the image plane and the source plane, clearly show that SGAS 1438 is dominated by a disk with an exponential light distribution. This is consistent with the visual impression of the system as a two arm "grand design" spiral galaxy. The nucleus of SGAS 1438 also appears significantly elongated, suggesting a bar.

\subsection{Color and Color Gradients}

Figures 6 and 7 include both color composite images as well as grayscale images reconstructed in the source plane in various filters. The images show that SGAS 1438 has an overall red and uniform morphology. The most significant deviations from color uniformity occurs in one of the putative spiral arms, which shows a few bluer clumps. Note that only the core of SGAS 1438 was targeted for near-IR spectroscopy and shown to have vigorous star formation - the uniform red light seen across much of the source is that of a vigorously star forming disk. A possible alternative interpretation of the source is as a merger with both arms representing tidal tails; blue tidal features with many young stars are seen in mergers nearby (e.g., Whitmore et al. 2010). 
However, the good fit to an exponential disk model, and the otherwise uniform colors of this source disfavor the merger hypothesis. Higher resolution imagery will be required to further illuminate this question.

\section{DISCUSSION AND CONCLUSIONS}

The far-IR luminosity of SGAS 1438 makes it a "Luminous Infrared Galaxy," or LIRG. It is instructive to place SGAS 1438 in the context of the deep Spitzer surveys performed using the MIPS $24 \mu \mathrm{m}$ band. The majority of galaxies detected in those surveys have redshifts of $0.6<z<1.2$ (Le Floc'h et al. 2005). At $z=0.8$, the typical infrared luminosity for MIPS detections is $L(\mathrm{TIR}) \sim 1 \times 10^{11} L_{\odot}$, with uncertainties of the order of a factor of two to three (Le Floc'h et al. 2005). The redshift and luminosity of SGAS 1438 of $z=0.816$ and $L$ (TIR) $=4.0 \times 10^{11} L_{\odot}$ mean that if SGAS 1438 was not lensed, then it would appear as a rather typical actively star-forming galaxy in the Spitzer deep fields. Such galaxies dominate the SFR density of the universe at that epoch (Papovich et al. 2004; Le Floc'h et al. 2005)

Rujopakarn et al. (2011) succinctly summarize results from the past decade, showing that actively star-forming galaxies in the distant universe are different from $z=0$ analogues of comparable luminosity in at least three major ways. First, their infrared SEDs are colder. Second, their aromatic features grow stronger with redshift for fixed $L$ (TIR). Third, their star formation occurs on physically larger scales.

The first two effects were discovered first, and a common cause was suspected: if star formation was happening on large spatial scales, that would naturally explain the higher aromatic feature strengths (less extinction) and colder SEDs (less intense radiation fields). New measurements of large sizes for $z>1$ LIRGs and ULIRGs (Rujopakarn et al. 2011) confirm those predictions. Thus, we have a framework to understand how the population of vigorously star-forming galaxies evolve with redshift. Though SGAS 1438 is only one galaxy, its high lensing magnification enables us to test this framework at otherwise unavailable photometric quality and spatial resolution. Thus, we now summarize the evidence for evolution with redshift in the SED shape, aromatic strength, and size of star-forming regions in luminous infrared galaxies, and discuss how SGAS 1438 tests this picture.

Cooler SEDs. That luminous infrared galaxies in the distant universe may have colder $\operatorname{SEDs}^{19}$ than at $z=0$ was suggested by Efstathiou \& Rowan-Robinson (2003) and first measured by Rowan-Robinson et al. (2004) from ISO data for LIRGs at $0.15<z<0.5$, and then confirmed with Spitzer (RowanRobinson et al. 2005; Sajina et al. 2006; Symeonidis et al. 2009). Spitzer 70 and $160 \mu \mathrm{m}$ photometry showed that LIRGs and ULIRGs at $z \sim 1$ have, on average, colder far-infrared SEDs that peak at longer wavelengths than $z \sim 0$ analogues (Symeonidis et al. 2009). Figure 5 of Symeonidis et al. (2009) illustrates how $0.1<z<1$ LIRGs have longer-wavelength SED peaks (in $f_{v}$ ) than $z=0$ LIRGs. The SED peak of SGAS 1438 is entirely consistent with their distant LIRG sample, and inconsistent with the $z=0$ sample. Those authors note that a simple way to describe the evolution in the luminosity-SED

\footnotetext{
19 As is typical in the U/LIRG literature, we use the words "hotter" and "cooler" SEDs as a shorthand, meaning that the peak of the far-IR spectral energy distribution is shifted to shorter wavelengths or longer wavelengths, respectively.
}

relation with redshift is that "the far-IR SEDs of many highredshift sources may resemble more closely those of lowerluminosity galaxies locally." This result was better quantified with multiband photometry from Herschel: Elbaz et al. (2010) and Hwang et al. (2010) found that the median dust temperature of LIRGs at $z \sim 1$ is several K colder than $z=0$ analogues; the offset is even more pronounced at higher luminosities.

The dust-reprocessed SED of SGAS 1438 is entirely consistent with these trends. We fit the SED with the local template set of Rieke et al. (2009), with the luminosity scaling as a free parameter. The best-fitting template belongs to $z=0$ galaxies that are nearly an order of magnitude less IR-luminous than SGAS 1438; that template peaks in $f_{v}$ at $130 \mu \mathrm{m}$. By contrast, the Rieke templates that bracket the observed $L$ (TIR) luminosity of SGAS 1438 have $f_{v}$ peaks at 75 to $80 \mu \mathrm{m}$. Thus, the dust SED of SGAS 1438 looks like a cool, low-luminosity galaxy that has been "scaled up" in luminosity.

Aromatic feature strength. The evolution toward stronger aromatic features with redshift, at fixed $L(T I R)$, was first discovered from Spitzer mid-infrared spectra and far-IR SEDs of $1<z<3$ galaxies (Papovich et al. 2007; Rigby et al. 2008; Farrah et al. 2008; Menéndez-Delmestre et al. 2009). The explanation proposed by the latter three sets of authors was that star formation in distant galaxies may occur on larger spatial scales than in local galaxies. Unfortunately, for SGAS 1438, we cannot isolate the aromatic feature strengths, as we lack mid-infrared spectra for this source. The WISE photometry at 12 and $22 \mu \mathrm{m}$ does contain flux from the aromatic features, but blended in an unknown ratio with hot dust continuum. Thus, it is not possible to test whether the aromatic features in SGAS 1438 obey the evolutionary relation noted in Rigby et al. (2008).

Size. Rujopakarn et al. (2011) measured Gaussian sizes of $0.4<z<2.5$ LIRGs and ULIRGs of $2-10 \mathrm{kpc}$. These are considerably larger than the measured $\lesssim 1 \mathrm{kpc}$ sizes of star formation in comparable-luminosity galaxies at $z=0$. Thus, Rujopakarn et al. (2011) conclude that luminous star formation episodes at $z=0$ are found only on sub-galactic $(D<1 \mathrm{kpc})$ spatial scales, while at $z>0.4$ they occur on larger, galaxy-wide scales $(D \sim 3-8 \mathrm{kpc}$ ), with consequently lower SFR surface densities.

The Gaussian size of SGAS $1438(7.4 \pm 0.6 \mathrm{kpc})$ is slightly larger than the sizes of 3-6 kpc measured by Rujopakarn et al. (2011) for $z \sim 1$ LIRGs, but consistent given our uncertainties and their small sample size. We can also compare to size measurements from Hubble Space Telescope imaging of disk galaxies in the GEMS fields. Barden et al. (2005) predict a relationship between effective radius (of a Sérsic profile fit) and total stellar mass. For the measured stellar mass of SGAS 1438 of $\sim 6 \times 10^{10} M_{\odot}$, the predicted effective radius from their mean relation is $\sim 7 \mathrm{kpc}$. This is somewhat larger than the measured value of $4.3 \pm 0.4 \mathrm{kpc}$ for SGAS 1438 , though consistent with expectations given the significant spread in the values of effective radius at fixed stellar mass apparent in the results of Barden et al. (2005).

SGAS 1438 in this Context. SGAS 1438 is only a single galaxy, but the strong lensing magnification allows for a detailed suite of measurements otherwise impossible to obtain. The redshift, luminosity, and far-IR SED of SGAS 1438 show it to be a typical vigorously star forming galaxy at $z \sim 0.8$, and thus representative of those galaxies that contribute most of the universe's star formation at this earlier epoch. (Its dustreprocessed SED, which we measure at high signal-to-noiseratio, is clearly cooler than comparably luminous galaxies at 
$z=0$, as are its peers at similar redshift). The spatial extent of star formation in SGAS 1438 is much larger than in comparably luminous galaxies at $z=0$, and comparable to (or slightly larger than) the half-dozen LIRGs measured at $0.5<z<1$ by Rujopakarn et al. (2011), confirming that result at higher signalto-noise and spatial resolution. The observations of SGAS 1438 presented here knit together both the size and SED-toluminosity evolution seen piecemeal in other work in a single, and apparently typical, object.

What is unique about SGAS 1438 is that lensing magnification allows us to determine the morphology of this vigorous star formation at high spatial resolution and signal-to-noise ratio. Morphological measurements, both in the image plane and the reconstructed source plane, strongly suggest that the star formation in SGAS 1438 is occurring in a large exponential disk. The surprising result, as shown in Figure 7, is that star formation appears to be distributed reasonably smoothly over the entire disk of this galaxy.

Thus, in this single but typical object, by robustly measuring a size, a dust temperature, and a total infrared luminosity, we confirm the picture from previous work that star formation in luminous infrared galaxies at $z=1$ occurs on much larger physical scales than in comparable-luminosity galaxies at $z$ $=0$. Furthermore, at high spatial resolution in SGAS 1438, we see that star formation is taking place smoothly across an exponential disk with an effective radius of $4.3 \pm 0.6 \mathrm{kpc}$ and an appearance suggestive of a two-armed "grand design" spiral morphology. The only somewhat comparable object in the local volume is HIZOA J0836-43 (Cluver et al. 2008, 2010) -an LIRG that similarly shows a large disk, a cool FIR SED, and an SFR of $\sim 21 M_{\odot} \mathrm{yr}^{-1}$; HIZOA J0836-43 is extremely gas $\operatorname{rich}\left(M_{\mathrm{HI}}=7.5 \times 10^{10} M_{\odot}\right.$ : Cluver et al. 2008), suggesting that SGAS 1438 may be an excellent target for future observations of molecular gas content, for example with ALMA or the EVLA.

L.A. thanks Alan Dressler for extensive discussions regarding velocity dispersion measurements and for kindly providing spectra on Virgo early-type galaxies. We thank the Herschel Science Centre for Director's Discretionary time to observe SGAS 1438.

M.D.G. thanks the Research Corporation for support of this work through a Cottrell Scholars award.

This publication makes use of data products from the Widefield Infrared Survey Explorer, which is a joint project of the University of California, Los Angeles, and the Jet Propulsion Laboratory/California Institute of Technology, funded by the National Aeronautics and Space Administration (NASA).

This work made use of observations made with the Spitzer Space Telescope, which is operated by the Jet Propulsion Laboratory, California Institute of Technology under a contract with NASA. Partial support for this work was provided by NASA through an award issued by JPL/Caltech.

This work includes observations obtained at the Gemini Observatory, which is operated by the Association of Universities for Research in Astronomy, Inc., under a cooperative agreement with the National Science Foundation (NSF) on behalf of the Gemini partnership: the NSF (United States), the Science and Technology Facilities Council (United Kingdom), the National Research Council (Canada), CONICYT (Chile), the Australian Research Council (Australia), Ministrio da Cincia, Tecnologia e Inovao (Brazil), and Ministerio de Ciencia, Tecnologa e Innovacin Productiva (Argentina).
This work was partially supported by a NASA Keck PI Data Award, administered by the NASA Exoplanet Science Institute. This work includes data obtained at the W. M. Keck Observatory from telescope time allocated to NASA through the agency's scientific partnership with the California Institute of Technology and the University of California. The Observatory was made possible by the generous financial support of the W. M. Keck Foundation.

This publication makes use of data products from the Two Micron All Sky Survey, which is a joint project of the University of Massachusetts and the Infrared Processing and Analysis Center/California Institute of Technology, funded by NASA and the NSF.

We acknowledge the use of data from the UVES Paranal Observatory Project (ESO DDT Program ID 266.D-5655).

This paper makes use of the ROSAT Data Archive of the MaxPlanck-Institut für extraterrestrische Physik (MPE) at Garching, Germany.

The authors acknowledge the very significant cultural role and reverence that the summit of Mauna Kea has always had within the indigenous Hawaiian community. We are most fortunate to have the opportunity to conduct observations from this mountain.

\section{APPENDIX}

\section{THE RED HERRING}

The discovery of cluster SDSSJ $1438+1454$ as a strong lens has a curious history, in that the initially identified arc candidate proved not to be lensed at all, while the follow-up infrared imagery revealed a different, very red galaxy that was also suggested in the SDSS imaging, is actually lensed, and is the subject of this paper. For completeness, we now describe the initial arc candidate.

The lensing search process detailed in M. D. Gladders et al. (in preparation) does not record which feature caused a particular field to be flagged as a candidate for lensing; the most likely feature is this arclet-like source, though a second faint and red feature - the lensed source discussed in this paper-is just visible in the SDSS imaging. A portion of the custom SDSS color image used in the lensing search of Gladders et al. is reproduced in Figure 1.

Follow-up imaging from the Nordic Optical Telescope (Figure 1) strengthened the lensing interpretation for the candidate arclet, since the curvature suggested in the SDSS imaging is confirmed, and the image does not show any bulge-like component that might be expected in the case of an edge-on galaxy.

The candidate blue arclet was also observed spectroscopically using the ARC $3.5 \mathrm{~m}$ telescope and the DIS on 2008 March 2. These data show that the candidate blue arclet is not in fact lensed; rather, it has a redshift of $z=0.231$ from clear detections of [O II] 3727 and $\mathrm{H} \alpha$, which is consistent with this source being a cluster member, given the SDSS redshift for a nearby apparent cluster galaxy at $z=0.237$ as well as the redshift of the BCG of 0.2373 (see Section 4.1).

\section{REFERENCES}

Allam, S. S., Tucker, D. L., Lin, H., et al. 2007, ApJL, 662, L51 Asplund, M., Grevesse, N., Sauval, A. J., \& Scott, P. 2009, ARA\&A, 47, 481 Bagnulo, S., Jehin, E., Ledoux, C., et al. 2003, Msngr, 114, 10 Barden, M., Rix, H.-W., Somerville, R. S., et al. 2005, ApJ, 635, 959 Bayliss, M. B. 2012, ApJ, 744, 156

Bayliss, M. B., Gladders, M. D., Oguri, M., et al. 2011, ApJL, 727, L26 
Bayliss, M. B., Wuyts, E., Sharon, K., et al. 2010, ApJ, 720, 1559 Becker, M. R., McKay, T. A., Koester, B., et al. 2007, ApJ, 669, 905 Becker, R. H., White, R. L., \& Helfand, D. J. 1995, ApJ, 450, 559 Belokurov, V., Evans, N. W., Moiseev, A., et al. 2007, ApJL, 671, L9 Bolzonella, M., Miralles, J.-M., \& Pelló, R. 2000, A\&A, 363, 476 Borys, C., Chapman, S., Donahue, M., et al. 2004, MNRAS, 352, 759 Bruzual, G., \& Charlot, S. 2003, MNRAS, 344, 1000

Calzetti, D., Armus, L., Bohlin, R., et al. 2000, ApJ, 533, 682

Caputi, K. I., Lagache, G., Yan, L., et al. 2007, ApJ, 660, 97

Chabrier, G. 2003, PASP, 115, 763

Combes, F., Rex, M., Rawle, T. D., et al. 2012, A\&A, 538, L4

Cluver, M. E., Jarrett, T. H., Appleton, P. N., et al. 2008, ApJL, 686, L17

Cluver, M. E., Jarrett, T. H., Kraan-Korteweg, R. C., et al. 2010, ApJ, 725, 1550

Dressler, A. 1984, ApJ, 281, 512

Efstathiou, A., \& Rowan-Robinson, M. 2003, MNRAS, 343, 322

Egami, E., Misselt, K. A., Rieke, G. H., et al. 2006, ApJ, 647, 922

Egami, E., Rex, M., Rawle, T. D., et al. 2010, A\&A, 518, L12

Elbaz, D., Hwang, H. S., Magnelli, B., et al. 2010, A\&A, 518, L29

Elíasdóttir, Á., Limousin, M., Richard, J., et al. 2007, arXiv:0710.5636

Farrah, D., Lonsdale, C. J., Weedman, D. W., et al. 2008, ApJ, 677, 957

Frayer, D. T., Harris, A. I., Baker, A. J., et al. 2011, ApJL, 726, L22

Fu, H., Jullo, E., Cooray, A., et al. 2012, ApJ, 753, 134

González-Nuevo, J., Lapi, A., Fleuren, S., et al. 2012, ApJ, 749, 65

Greve, T. R., Vieira, J. D., Weiß, A., et al. 2012, ApJ, 756, 101

Harris, A. I., Baker, A. J., Frayer, D. T., et al. 2012, ApJ, 752, 152

Hao, J., McKay, T. A., Koester, B. P., et al. 2010, ApJS, 191, 254

Hezaveh, Y. D., Marrone, D. P., \& Holder, G. P. 2012, ApJ, 761, 20

Hwang, H. S., Elbaz, D., Magdis, G., et al. 2010, MNRAS, 409, 75

Jullo, E., Kneib, J.-P., Limousin, M., et al. 2007, NJPh, 9, 447

Kelson, D. D., Illingworth, G. D., van Dokkum, P. G., \& Franx, M. 2000, ApJ, 531,159

Kennicutt, R. C., Jr. 1998, ApJ, 498, 541

Kneib, J.-P., van der Werf, P. P., Kraiberg Knudsen, K., et al. 2004, MNRAS, 349,1211

Knudsen, K. K., Neri, R., Kneib, J.-P., \& van der Werf, P. P. 2009, A\&A, 496, 45

Koester, B. P., Gladders, M. D., Hennawi, J. F., et al. 2010, ApJL, 723, L73

Lagache, G., Puget, J.-L., \& Dole, H. 2005, ARA\&A, 43, 727

Le Floc'h, E., Papovich, C., Dole, H., et al. 2005, ApJ, 632, 169

Lestrade, J.-F., Carilli, C. L., Thanjavur, K., et al. 2011, ApJL, 739, L30

Limousin, M., Kneib, J.-P., \& Natarajan, P. 2005, MNRAS, 356, 309

Lin, H., Buckley-Geer, E., Allam, S. S., et al. 2009, ApJ, 699, 1242

Markwardt, C. B. 2009, in ASP Conf. Ser. 411, Astronomical Data Analysis Software and Systems XVIII, ed. D. A. Bohlender, D. Durand, \& P. Dowler (San Francisco, CA: ASP), 251
Menéndez-Delmestre, K., Blain, A. W., Smail, I., et al. 2009, ApJ 699,667

Negrello, M., Hopwood, R., De Zotti, G., et al. 2010, Sci, 330, 800

Niederste-Ostholt, M., Strauss, M. A., Dong, F., Koester, B. P., \& McKay, T. A. 2010, MNRAS, 405, 2023

Oke, J. B. 1974, ApJS, 27, 21

Papovich, C., Dole, H., Egami, E., et al. 2004, ApJS, 154, 70

Papovich, C., Rudnick, G., Le Floc'h, E., et al. 2007, ApJ, 668, 45

Pascale, E., Ade, P. A. R., Bock, J. J., et al. 2009, ApJ, 707, 1740

Peng, C. Y., Ho, L. C., Impey, C. D., \& Rix, H.-W. 2010, AJ, 139, 2097

Pettini, M., \& Pagel, B. E. J. 2004, MNRAS, 348, L59

Rieke, G. H., Alonso-Herrero, A., Weiner, B. J., et al. 2009, ApJ, 692,556

Rigby, J. R., Marcillac, D., Egami, E., et al. 2008, ApJ, 675, 262

Rigby, J. R., Wuyts, E., Gladders, M. D., Sharon, K., \& Becker, G. D. 2011, ApJ, 732,59

Rowan-Robinson, M., Babbedge, T., Surace, J., et al. 2005, AJ, 129,1183

Rowan-Robinson, M., Lari, C., Perez-Fournon, I., et al. 2004, MNRAS, 351, 1290

Rujopakarn, W., Rieke, G. H., Eisenstein, D. J., \& Juneau, S. 2011, ApJ, 726,93

Rujopakarn, W., Rieke, G. H., Weiner, B. J., et al. 2011, ApJ, submitted (arXiv: 1107.2921R)

Sajina, A., Scott, D., Dennefeld, M., et al. 2006, MNRAS, 369, 939

Sharon, K., Gladders, M. D., Rigby, J. R., et al. 2012, ApJ, 746, 161

Siana, B., Smail, I., Swinbank, A. M., et al. 2009, ApJ, 698, 1273

Skrutskie, M. F., Cutri, R. M., Stiening, R., et al. 2006, AJ, 131, 1163

Smail, I., Ivison, R. J., \& Blain, A. W. 1997, ApJL, 490, L5

Smail, I., Swinbank, A. M., Richard, J., et al. 2007, ApJL, 654, L33

Spitler, L. R., Labbé, I., Glazebrook, K., et al. 2011, ApJL, 748, L21

Storey, P. J., \& Zeippen, C. J. 2000, MNRAS, 312, 813

Swinbank, A. M., Smail, I., Longmore, S., et al. 2010, Natur, 464, 733

Symeonidis, M., Page, M. J., Seymour, N., et al. 2009, MNRAS, 397, 1728

Vieira, J. D., Crawford, T. M., Switzer, E. R., et al. 2010, ApJ, 719, 763

Wardlow, J. L., Cooray, A., De Bernardis, F., et al. 2012, ApJ, 762, 59

Whitmore, B. C., Chandar, R., Schweizer, F., et al. 2010, AJ, 140, 75

Williams, L. L. R., \& Lewis, G. F. 1996, MNRAS, 281, L35

Wright, E. L., Eisenhardt, P. R. M., Mainzer, A. K., et al. 2010, AJ, 140,1868

Wuyts, E., Barrientos, L. F., Gladders, M. D., et al. 2010, ApJ, 724, 1182

Wuyts, E., Rigby, J. R., Gladders, M. D., et al. 2012, ApJ, 745, 86

Yee, H. K. C., Ellingson, E., Bechtold, J., Carlberg, R. G., \& Cuillandre, J.-C. 1996, AJ, 111, 1783 